Florida International University FIU Digital Commons

6-12-2015

\title{
Open Trial and Pilot Randomized Controlled Trial of a Novel Program to Reduce Perceived Burdensomeness
}

Ryan M. Hill

Florida International University, rhillo04@fiu.edu

DOI: $10.25148 /$ etd.FIDC000057

Follow this and additional works at: https:// digitalcommons.fiu.edu/etd

Part of the Child Psychology Commons, and the Clinical Psychology Commons

\section{Recommended Citation}

Hill, Ryan M., "Open Trial and Pilot Randomized Controlled Trial of a Novel Program to Reduce Perceived Burdensomeness" (2015). FIU Electronic Theses and Dissertations. 2243.

https://digitalcommons.fiu.edu/etd/2243 


\title{
FLORIDA INTERNATIONAL UNIVERSITY
}

Miami, Florida

\section{OPEN TRIAL AND PILOT RANDOMIZED CONTROLLED TRIAL OF A NOVEL PROGRAM TO REDUCE PERCEIVED BURDENSOMENESS}

\author{
A dissertation submitted in partial fulfillment of the \\ requirements for the degree of \\ DOCTOR OF PHILOSOPHY \\ in \\ PSYCHOLOGY
}

by

Ryan Michael Hill

2015 
To: Dean Michael R. Heithaus

College of Arts and Sciences

This dissertation, written by Ryan Michael Hill, and entitled Open Trial and Pilot Randomized Controlled Trial of a Novel Program to Reduce Perceived Burdensomeness, having been approved in respect to style and intellectual content, is referred to you for judgment.

We have read this dissertation and recommend that it be approved.

Daniel Bagner

Raul Gonzalez, Jr.

Maureen Kenny

Jeremy Pettit, Major Professor

Date of Defense: June 12, 2015

The dissertation of Ryan Michael Hill is approved.

\begin{tabular}{r}
\hline $\begin{array}{r}\text { Dean Michael R. Heithaus } \\
\text { College of Arts and Sciences }\end{array}$ \\
\hline Dean Lakshmi N. Reddi \\
University Graduate School
\end{tabular}

Florida International University, 2015 
C Copyright 2015 by Ryan Michael Hill

All rights reserved. 


\section{ACKNOWLEDGMENTS}

To the mentors who have provided guidance and support, the instructors who have opened doors and showed me new possibilities, and to the friends and colleagues who have encouraged, put up with, and artfully distracted me: You each have my profound thanks.

This work would not have been possible, first and foremost, without the support of my faculty advisor, Dr. Jeremy Pettit, my dissertation committee members, and the faculty who have provided support, both formal and informal, along the way. Nor would this work have been completed without the assistance of my fellow graduate students and a dedicated team of research assistants.

Finally, this work was made possible by the support of a Florida International University Presidential Fellowship Award, Florida International University Doctoral Evidence Acquisition Award, and a 2014 American Psychological Foundation Visionary Funds Grant. 


\title{
ABSTRACT OF THE DISSERTATION \\ OPEN TRIAL AND PILOT RANDOMIZED CONTROLLED TRIAL OF A NOVEL PROGRAM TO REDUCE PERCEIVED BURDENSOMENESS
}

\author{
by \\ Ryan Michael Hill \\ Florida International University, 2015 \\ Miami, Florida \\ Professor Jeremy Pettit, Major Professor
}

To date, suicide prevention programs for adolescents have not demonstrated sustained reductions in suicide-related behaviors and further program development is called for, particularly for the prevention of non-clinical suicide risk. This research utilizes the Interpersonal-Psychological Theory of Suicide in the development of the LEAP Program, a novel, web-based suicide prevention program targeting reductions in cognitions of perceived burdensomeness. An open trial of the program was conducted to examine the feasibility of the study protocol, generate feedback regarding the LEAP program modules, and refine the program modules. A pilot randomized controlled trial of the program was also conducted to examine participant satisfaction with the intervention and adherence to the intervention protocol, to test the research protocol, and to provide initial evidence for its efficacy. The open trial consisted of eight adolescents who completed a baseline assessment, received the LEAP intervention, and completed a post-intervention assessment. Results indicated sufficient feasibility of the study protocol and acceptability of the LEAP intervention. The pilot randomized controlled trial consisted of 80 adolescents who were randomly assigned to either the LEAP intervention or a treatment- 
as-usual control condition. Results indicated that those who completed the LEAP intervention showed significantly reduced perceived burdensomeness scores at postintervention, as compared to those in the control condition. In addition, those who completed the intervention reported significantly reduced perceived burdensomeness, thwarted belongingness, and depressive symptom scores at follow-up, as compared to those in the control condition. No significant reductions in suicidal ideation were noted for those who completed the intervention, as compared to those in the control condition. Strengths and weaknesses of the present studies are discussed, and considerations for future research directions are noted. 


\section{TABLE OF CONTENTS}

CHAPTER

PAGE

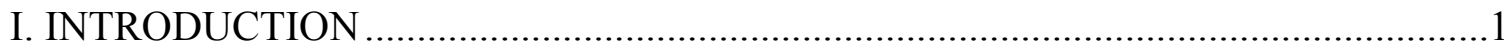

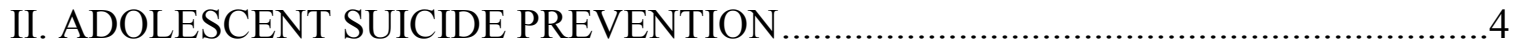

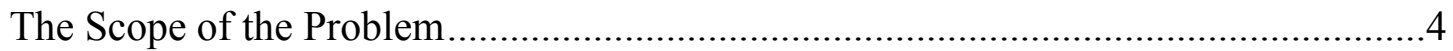

Current Approaches to Adolescent Suicide Prevention..............................................

A Way Forward for Developing Suicide Prevention Programs....................................22

III. SUICIDAL IDEATION: FROM A THEORETICAL MODEL TO A NOVEL

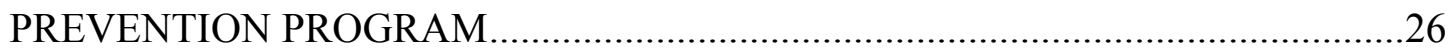

The Interpersonal-Psychological Theory of Suicide..................................................26

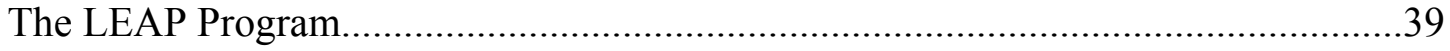

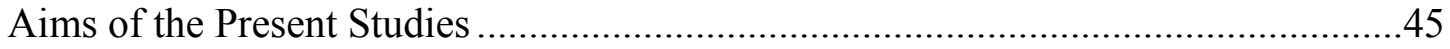

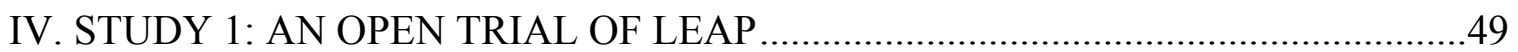

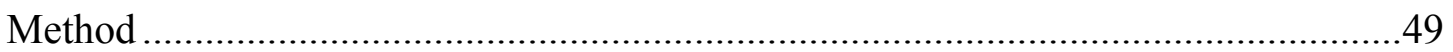

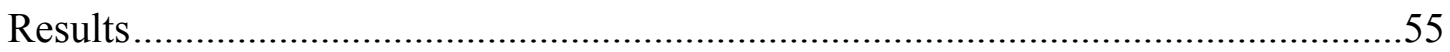

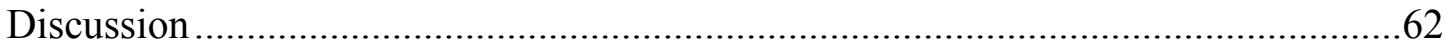

V. STUDY 2: A PILOT RANDOMIZED CONTROLLED TRIAL OF LEAP.................69

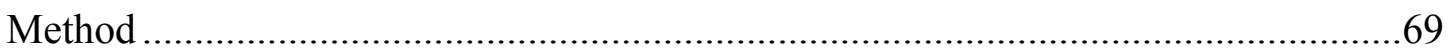

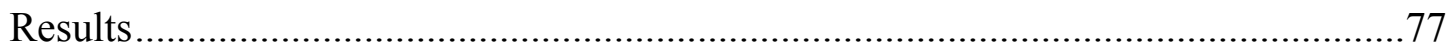

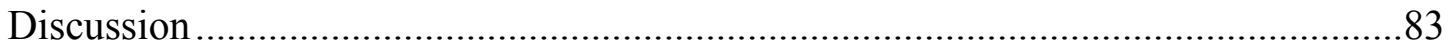

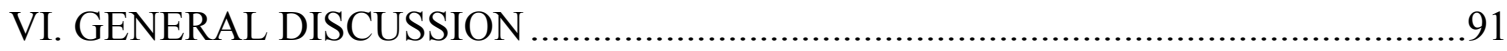

Summary of the Present Work ………………………......................................91

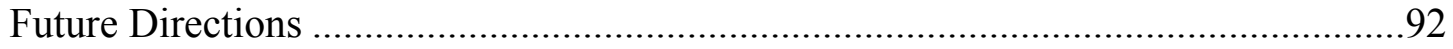

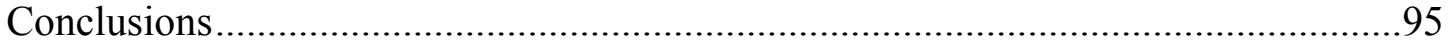

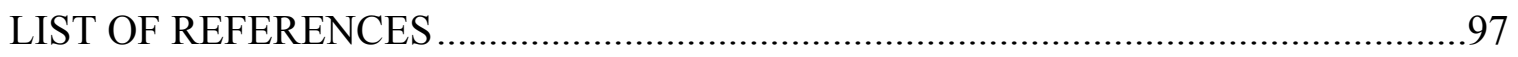

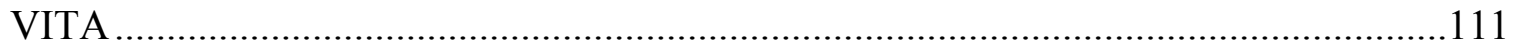




\section{LIST OF TABLES}

CHAPTER

PAGE

1. Summary of Adolescent Suicide Prevention Programs ..........................................21

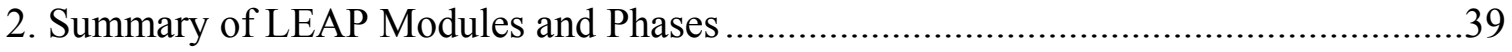

3. Means and Standard Deviations of Open Trial Study Measures .................................62

4. Summary of Modifications to the LEAP Modules ...................................................66

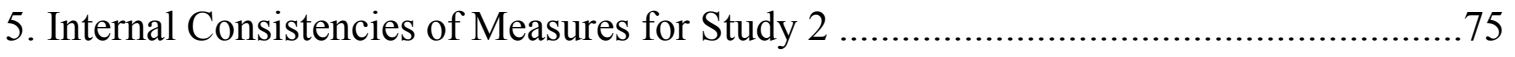

6. Demographic Characteristics of ITT, TxComplete, and Control Groups.....................78

7. Means and Standard Deviations of Pilot Randomized Controlled Trial Study

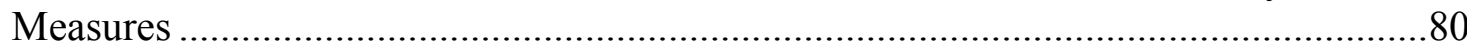




\section{LIST OF FIGURES}

FIGURE

PAGE

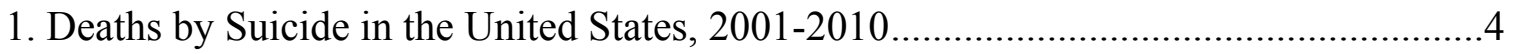

2. The Interpersonal-Psychological Theory of Suicide .............................................27

3. Learn Phase: The Affect-Behavior-Cognition Triangle .........................................41

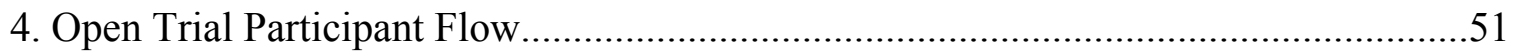

5. Pilot Randomized Controlled Trial Participant Flow ................................................ 74 


\section{INTRODUCTION}

Suicide is the third leading cause of death among 15-24 year olds in the United States (Centers for Disease Control and Prevention, 2014). Data from the Youth Risk Behavior Survey (Centers for Disease Control and Prevention, 2012) indicate that in the 12 months preceding the survey, $15.8 \%$ of high school students experienced serious suicidal ideation. Further development of prevention programs to reduce suicide-related behaviors, especially those focusing on non-clinical suicide risk management, is needed.

To date, most adolescent suicide prevention programs have focused either on universal approaches such as case identification or increasing public knowledge about warning signs of suicide (e.g., Wyman et al., 2008) or indicated approaches to reduce risk in adolescents reporting serious suicidal ideation or suicide attempts (King et al., 2009). These indicated programs typically focus on risk factor reduction, often targeting skills training and social support (e.g., Eggert, Thompson, Herting, \& Nicholas, 1995; King et al., 2006; Thompson, Eggert, Randell, \& Pike, 2001). While these programs represent promising first steps, they have not demonstrated sustained reductions in suicide-related behaviors (e.g., King, et al., 2009; King, et al., 2006; Wyman et al., 2010) and further program development is called for. A review of the extant literature on the state of prevention science for adolescent suicide-related behaviors leads to the identification of (a) relatively few indicated approaches to suicide prevention as well as (b) a number of issues related to the development of prevention programs for suicide-related behaviors. Issues include challenges in transporting evidence-based interventions to community settings (Kazdin \& Blasé, 2011), difficulties with sustainability of programs, barriers to treatment, and issues with portability of interventions to community settings. Potential 
solutions are presented, leading to the development of a novel suicide prevention program aimed at implementing these alternative strategies to prevention.

Namely, the present research utilizes the Interpersonal-Psychological Theory of Suicide (IPTS; Joiner, 2005) in the development of a suicide prevention program. The IPTS provides a theoretical account of suicide-related behaviors, including hypotheses that identify the circumstances in which suicidal ideation should be most likely to occur. The IPTS proposes that two factors are necessary for an individual to die by suicide, neither of which, alone, is sufficient: the acquired capability to enact lethal self-injury and the desire for death (Joiner, 2005). The acquired capability is viewed as a learned capability acquired via repeated exposure to pain and injury. It is a static risk factor and thus is not an efficient target for preventive interventions. The desire for death is roughly equivalent to the common definition of suicidal ideation (Van Orden, Witte, Gordon, Bender, \& Joiner, 2008). It is an individual's subjective desire to cease living. The desire for death is comprised of two factors: a sense of perceived burdensomeness (e.g., "My life is a drain on others") and thwarted belongingness (e.g., "There is nobody I can turn to"). Both are subjective perceptions of current life states, rather than stable conditions, and should be amenable to change. A review of the literature testing the hypotheses of the IPTS reveals strong support for a link between perceived burdensomeness and suicidal ideation, suggesting that reductions in perceived burdensomeness may potentially lead to reductions in suicidal ideation, though this hypothesis has not yet been subjected to empirical examination. Furthermore, most suicide prevention research has addressed thwarted belongingness-related factors such as connectedness and social support and has failed to show sustained reductions in suicidal ideation. Thus, the present studies leverage 
the IPTS, and perceived burdensomeness specifically, in a novel prevention program to reduce or prevent suicidal ideation among adolescents.

The present work begins with a review of the existing prevention literature and a review of the IPTS, which provided a catalyst for the creation of a novel prevention program. Then the development of a selected computer-based prevention program to reduce perceived burdensomeness (the LEAP program) is described. An open trial of the program was conducted to examine the feasibility of the study protocol, generate feedback regarding the LEAP program modules, and refine the program modules. Finally, a pilot randomized controlled trial of the program was conducted to examine participant satisfaction with the intervention and adherence to the intervention protocol, to test the research protocol, and to provide initial evidence for its efficacy. 


\section{ADOLESCENT SUICIDE PREVENTION}

\section{The Scope of the Problem.}

Adolescent suicide-related behaviors are a significant health problem in the United States. Suicide is the third leading cause of death for individuals between the ages of 15-24 years (Centers for Disease Control and Prevention, 2014a). From 2000-2010, the suicide rate among adolescents ages 13-17 years was 4.34 per 100,000, accounting for more than 10,000 deaths over that period (Centers for Disease Control and Prevention, 2014a). The suicide rate among emerging adults is higher still, approximately 12.24 per 100,000. Even so, suicide is rare prior to the onset of adolescence, with a rate of suicide of 0.52 per 100,000 among children 10-12 years of age (Centers for Disease Control and Prevention, 201a). Thus, as presented in Figure 1, the rate of suicide increases sharply beginning with the onset of adolescence and remains elevated throughout adulthood.

Figure 1. Deaths by Suicide in the United States, 2001-2010

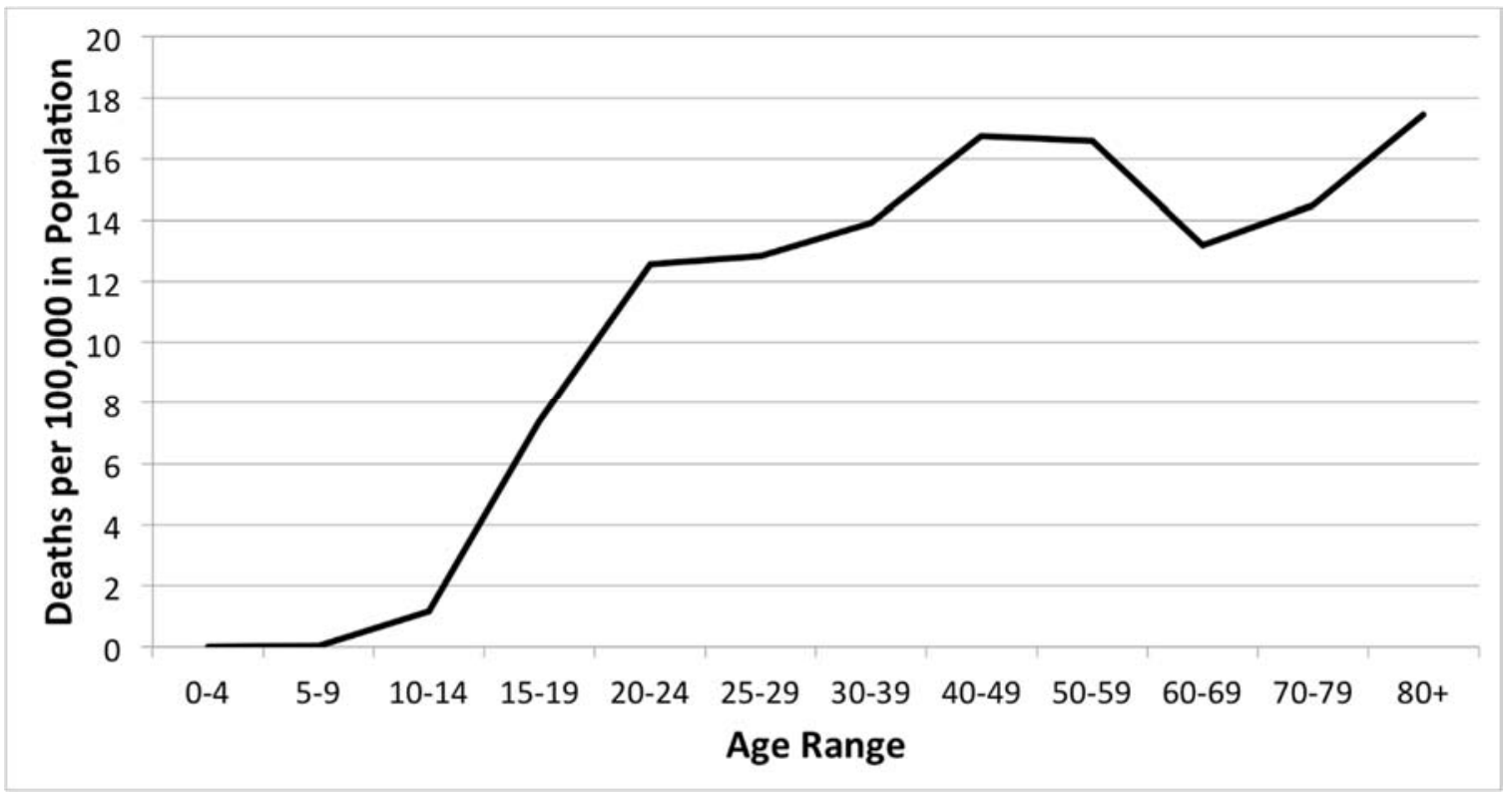


In addition to deaths by suicide, suicide attempts and suicidal ideation are also frequent during adolescence. Data from the Youth Risk Behavior Surveillance System, a nationally-representative survey of more than 15,000 United States high school students from over 158 schools, indicate that, in the previous 12 months, $17.0 \%$ of high school students seriously considered suicide, $13.6 \%$ made a suicide plan, and $8.0 \%$ made a suicide attempt (Centers for Disease Control and Prevention, 2014b). Data from the 2010 Minnesota Student Survey, a survey of more than $70,0009^{\text {th }}$ and $12^{\text {th }}$ grade students, reported similar findings with $13.5 \%$ of students reporting suicidal ideation or a suicide attempt in the past year (Taliaferro \& Muehlenkamp, 2014). The high rates of suicidal ideation and suicide attempts, coupled with the sharp increase in suicide deaths during this period, indicate a need to address suicide risk during the adolescent years. Efficacious suicide prevention efforts have the potential to substantially reduce this high number of preventable deaths. Further, the elevated rates of suicidal ideation and suicide attempts during adolescence indicate the enormity of the unaddressed mental health burden associated with suicide-related behaviors. Given that approximately $2.7 \%$ of adolescents report having made a suicide attempt requiring medical care in the previous 12 months (Centers for Disease Control and Prevention, 2014b), suicide-related behaviors also represent a substantial source of preventable burden on the medical care system in the United States.

Taken together, these data point toward adolescence as a key period for addressing suicide risk and demonstrate a need to reach this population with suicidefocused prevention programs. The National Strategy for Suicide Prevention (United States Department of Health and Human Services, 2001) and the 2010 Progress Review 
of the National Strategy for Suicide Prevention (Suicide Prevention Resource Center and SPAN USA, 2010) both declared suicide and suicide-related behaviors a national public health problem and recommended research to develop and evaluate effective therapies for clinical and non-clinical suicide risk. The United States Surgeon General's Call to Action to Prevent Suicide (United States Department of Health and Human Services, 1999) also included a call to develop and implement safe and effective programs to address adolescent suicide risk. Leaders within the field of suicidology have called for additional attention to be given to innovative strategies for prevention and early intervention of suicide-related behaviors (Asarnow \& Miranda, 2014).

For the sake of clarity, it is important to define the key terminology associated with suicide-related behaviors prior to reviewing the relevant literature: The currently accepted nomenclature in the field of suicidology identifies three distinct categories of suicide-related behaviors: suicide, nonfatal suicide attempts, and suicidal ideation (O'Carroll, Berman,Maris, \& Moscicki, 1996; Silverman, Berman, Sanddal, O'Carroll, \& Joiner, 2007). Suicidal ideation includes any self-reported thought of killing oneself, which may range from general thoughts of death and wishing to be dead to specific thoughts regarding suicide plans and making preparations for a suicide attempt. A suicide attempt is a non-fatal self-inflicted act where the individual has some intent to die and where there is the potential for injury, even where no serious injury occurs; and suicide is a fatal self-inflicted destructive act with some, non-zero explicit or implicit intent to die.

Researchers have called for a routine distinction between these three categories (Silverman et al., 2007), as they may be distinct phenomena, have differential risk 
factors, and because their prevalence rates differ markedly. Furthermore, these three categories together constitute "suicide-related behaviors," a more general term, sometimes also referred to as "suicidal thoughts and behaviors" or "suicidality" in the extant literature. As per the directive of the Centers for Disease Control and Prevention (Crosby, Ortega, \& Melanson, 2011), for the sake of clarity and to allow differentiation between the components of suicide-related behaviors, wherever possible in the review of the empirical literature, the specific components of suicide-related behaviors are referred to directly (suicide, suicide attempts, suicidal ideation). Where the distinction is not possible (e.g., where the method of measurement does not allow for differentiation between suicidal ideation and suicide attempts) the more general term of suicide-related behaviors is applied.

Although these three constructs are likely distinct, evidence demonstrates significant associations between suicide deaths among adolescents and previous suicidal ideation and suicide attempts (e.g., Beck, Steer, Kovacs, \& Garrison, 1985; Brent et al., 1993; Philips et al., 2002). By extension, the interrelation of these constructs of suiciderelated behaviors suggest that treatment and prevention of suicidal ideation has the potential to reduce more serious suicide-related behaviors.

\section{Current Approaches to Adolescent Suicide Prevention.}

Having established the need for additional prevention and intervention approaches aimed at reducing suicide-related behaviors in adolescents, it is important to first examine existing approaches to suicide prevention and their strengths and weaknesses. In developing novel approaches for suicide prevention, weaknesses in existing approaches 
should be addressed and efforts should be made to address avenues of prevention where existing programs have not proven efficacious.

Nearly two decades ago, the Summary of the Institute of Medicine Report on Prevention of Mental Disorders (Munoz, Mrazek, \& Haggerty, 1996) emphasized the need for a greater focus on "prevention services in settings other than mental health settings and on problems that transcend the usual definitions of mental disorders" ( $p$. 1117), a goal toward which suicide prevention researchers have made great efforts. The Institute of Medicine report also redefined prevention services within a larger spectrum of mental health interventions. As defined in the report, intervention for any mental health related issues include prevention, treatment, and maintenance phases.

The prevention phase includes any intervention that occurs prior to the onset of diagnosable disorders; the prevention phase was further subdivided into universal, selected, and indicated phases. Universal prevention focuses on broad, population-wide approaches within which targets are not identified on any criteria of increased risk. Wellknown examples of universal prevention strategies include the Drug Abuse Resistance Education (DARE) program to reduce drug use, seatbelts to prevent injury resulting from traffic collisions, and adding fluoride to drinking water to prevent tooth decay. Universal programs are applied to a population at-large, without regard to factors that may increase an individual's risk for the outcome in question. Selected prevention includes programs delivered to a subset of the general population deemed to be at increased risk of a negative outcome determined by some predefined risk factor. Examples of selected prevention programs might include depression prevention programs for teens of families in the process of a separation or divorce or providing the H1N1 influenza vaccine to 
medical professionals during the 2009 outbreak. Finally, indicated prevention includes programs that are directed toward individuals with detectable, subthreshold levels of a disorder that has not yet reached a diagnostic level. Here, examples include depression prevention programs for adolescents reporting sad mood, but falling below diagnostic criteria for Major Depressive Disorder or introducing a dieting intervention for overweight individuals in order to prevent obesity.

In terms of suicide prevention, universal prevention efforts have included population level efforts to distribute firearm safety information or increasing awareness of risk factors for suicide. Selected programs have included mentoring programs for adolescent bully victims/bully perpetrators and programs aimed at reducing suicide risk among sexual minority populations (e.g., The Trevor Project, 2015), which are at elevated risk for suicidal ideation (e.g., Hill \& Pettit, 2012), among others. Indicated programs, those that address suicidal thoughts in order to prevent suicide attempts and deaths by suicide, have included dialectical behavior therapy and multisystemic therapy, as well as programs aimed at increasing the social support networks of at-risk youths.

Of note, all programs designed to prevent suicide are, by definition, prevention programs. Those that address suicidal ideation or suicide attempts may also be viewed as treatment approaches for suicidal ideation or suicide attempts, respectively, under the rubric of the Institute of Medicine report, but these also serve the overall goal of suicide prevention and so will be treated as indicated prevention programs for the purpose of this review. Programs for those affected by the suicide of a loved one, what the field of suicidology most often terms "post-vention" are not suicide prevention programs per se, and will not be reviewed here. Often these programs include various support group and 
grief support models (see Jordan \& McMenamy, 2004), but are not specifically aimed at reducing future suicide-related behaviors.

The following sections review the literature on existing suicide prevention programs, roughly divided into universal, selected, and indicated approaches. A summary of existing suicide prevention programs for adolescents in presented in Table 1. (For a review of suicide prevention programs prior to 2003, see Gould, Greenberg, Velting, \& Shaffer, 2003; for additional reviews of suicide prevention, see Robinson, Hetrick, \& Martin, 2011 and van der Feltz-Cornelis et al., 2011.)

Universal prevention programs. Current universal approaches to suicide prevention among adolescents focus primarily on case identification via training gatekeepers (Isaac et al., 2009) and peer leaders to notice warning signs of suicide (Wyman et al., 2010). These universal approaches utilize psychoeducation modules to raise awareness, teach participants about risk factors for suicide, and increase participants' knowledge of appropriate action when encountering a suicidal individual (Isaac et al., 2009; Wyman et al., 2010; Zenere \& Lazarus, 1997).

The Sources of Strength program utilized adolescent peer leaders in high school settings to increase awareness of suicide risk factors and encourage students' helpseeking behavior. In one study, the Sources of Strength program resulted in significantly improved perceptions of adult help for adolescents with suicidal ideation, but did not demonstrate a reduction of suicide-related behaviors (Wyman et al., 2010). Other gatekeeper programs (for example, the Question, Persuade, Refer program; Quinnett, 1995), in which individuals are trained to recognize warning signs of, or risk factors for, suicide-related behaviors have been tested, though they often (a) train adult gatekeepers 
to recognize risk factors in adolescents or adult peers and (b) test for increases in knowledge or increases in comfort discussing suicidal thoughts, rather than reductions in reported suicide-related behaviors within an organization, school, or community (e.g., Ghoncheh, Kerkhof, \& Koot, 2014; Jacobson, Osteen, Sharpe, \& Pastoor, 2012; Wyman et al., 2008). Research supports the efficacy of these programs for increasing knowledge related to suicide risk factors and warning signs (see Isaac et al., 2009, for a review). Recent research has also supported the feasibility of a web-based gatekeeper training program (Lancaster et al., 2014). However, it remains unclear whether or not these prevention-through-awareness programs result in significant reductions in suicide-related behaviors within the organizations in which they have been implemented.

Of note, a more comprehensive universal prevention program than the one described above was implemented in the Dade County Public School system in the early 1990's. The program includes mental health promotion, organizational-level changes to coordinate suicide-prevention activities, and a brief psychoeducation unit about teenage suicide in the $10^{\text {th }}$ grade curriculum. The scope of the program was not amendable to randomized intervention research, but analysis of trends in suicide-related behaviors within the district before and after implementation of the program provided evidence of a decline in suicide attempts and suicide, but not suicidal ideation, following implementation of the program (Zenere \& Lazarus, 1997).

Selected prevention programs. Existing programs that fall in the domain of selected prevention include the Teen Options for Change (TOC; King, Gipson, \& Horwitz, 2014) and Links to Enhancing Teens' Connectedness (LET's CONNECT; Gipson, King, Opperman, \& Ewell-Foster, 2014) programs. Both focus on enhancing 
connectedness, which includes a feeling of relatedness and of being involved with other people or groups in a way that promotes a sense of well-being (Townsend \& McWhirter, 2005). Programs such as the It Gets Better campaign also fall under the heading of selected prevention, though such projects have not yet been subjected to empirical evaluation.

The TOC program attempts to connect adolescents at elevated risk for suiciderelated behaviors to mental health services via a brief, motivational interviewing based intervention (King, Gipson, \& Horwitz, 2014). Teen Options for Change identifies adolescents at elevated risk for suicide via a brief survey conducted within a medical emergency department. Motivational interviewing techniques are then used to help teens increase their willingness and desire to seek mental health treatment and therefore increase their likelihood of connecting to mental health services after discharge from the emergency department. A pilot RCT of this program has been completed and the program did not demonstrate significant reductions in suicidal ideation at follow-up, nor did it produce a significant increase in mental health service utilization when compared to a control group (King, Gipson, \& Horwitz, 2014).

The LET'S CONNECT program (Gipson et al., 2014), currently being examined in an RCT, identifies adolescents at risk for suicide-related behaviors on the basis of selfreported bully victimization, bully perpetration, and/or social disconnection from peers. The LET'S CONNECT program utilizes a mentorship model to increase teens' perceptions of social connection. Adolescents are identified via screening questionnaires in a medical emergency department. Those who are identified as being at risk and who enroll in the program are matched to a community mentor and identify a second "natural" 
mentor (an adult relative, friend of the family, etc.). Natural and community mentors both agree to a brief training session with a clinician and to engage in regular contact with the adolescent. Outcomes for the LET'S CONNECT program are not yet available.

Indicated prevention programs. Indicated approaches targeting connectedness include the Youth-Nominated Support Team (YST) I and II trials for psychiatrically hospitalized adolescents (King et al., 2006; King et al., 2009) and the EDge project, a postcard-based supportive intervention (Carter et al., 2007) currently being adapted for adolescents (Robinson et al., 2009). Additional interventions include the use of rapidresponse teams to link suicidal emergency department patients to outpatient services (Greenfield, Lawson, Hechtman, Rousseau, \& Platt, 2002; Latimer, Gariepy, \& Greenfield, 2014), dialectical behavior therapy (Katz, Cox, Gunasekara, \& Miller 2004; Rathus \& Miller, 2002), multisystemic therapy (Huey et al., 2004), skills-based treatment (Donadlson, Spirito, \& Esposito-Smythers, 2005), individual cognitive-behavioral therapy (Esposito-Smythers, Spirito, Kahler, Hunt, \& Monti, 2011), and crisis hotlines for urgent counseling (Gould, Cross, Pisani, Munfakh, \& Kleinman, 2013).

The YST I trial focused on developing the social networks of psychiatrically hospitalized suicidal youth. The adolescents, in conjunction with a therapist, identified four potential support persons who were then enrolled in the program and provided psychoeducation sessions regarding the youths' psychiatric issues. These support persons were then asked to maintain weekly contact with the adolescent to provide a base of social support. The YST I trial demonstrated no overall main effects for adolescentreported suicide-related behaviors, but did report reductions in adolescent-reported suicidal ideation among girls, but not among boys (King et al., 2006). The YST-II 
program, which similarly focused on enhancing adolescents' contact with supportive adults, reported more rapid declines in suicidal ideation among multiple suicide attempters than controls in the first six weeks after discharge from a psychiatric unit, but no enduring effects on suicidal ideation or suicide attempts (King et al., 2009). Both YST trials focused on developing the supportive networks of adolescents after a psychiatric hospitalization for a suicide attempt or suicidal crisis.

The EDge Project is a support post-card based intervention program. Adolescents who report to a specialist mental health service are sent monthly, supportive, personalized post cards for 12 months (Robinson et al., 2009). In a study among adults, the supportive post card condition did not lead to a significantly lower proportion of people selfpoisoning as compared to the control condition, but did lead to a lower mean number of self-poisoning episodes compared to the control group (Carter et al., 2007).

A trial in Canada examined the use of rapid response teams designed to identify adolescents reporting to the emergency department after a suicide attempt or during a suicidal crisis and link them to outpatient treatment services (Greenfield et al., 2002). Each rapid response team consisted of, at minimum, a psychiatrist and a psychiatric nurse who met with families within 72 hours of their emergency department visit to help link the adolescent to outpatient psychiatric or psychological services. In a quasi-randomized study, adolescents assigned to the rapid response team model of care were less likely to be subsequently hospitalized, as compared to adolescents who received usual care. However, there were no significant differences between the two groups with regard to level of functioning or suicide-related behaviors at the follow-up assessment (Greenfield et al., 2002). The rapid response model was more cost-effective as a method for linking 
suicidal adolescents to outpatient care as compared to usual care practices, as a result of the reduced rate of hospitalizations (Latimer, Gariepy, \& Greenfield, 2014).

Another treatment protocol, Dialectical Behavior Therapy for Adolescents (DBT) is included in the American Psychological Association Division 12's list of researchsupported psychological treatments (American Psychological Association, 2015) and is considered an intervention with "strong research support." Several treatment components comprise the DBT approach, including behavioral strategies, crisis intervention support, and mindfulness and acceptance-based strategies taught via both individual and group treatment sessions (American Psychological Association, 2015). Dialectical Behavior Therapy has established a base of empirical support with adults, including having demonstrated reductions in suicide attempts (Linehan et al., 2006).

Evidence supporting the use of DBT with adolescents has been somewhat mixed: Dialectical Behavior Therapy demonstrated within-group pre-post reductions in adolescent suicidal ideation in one study (Rathus \& Miller, 2002). That same study, however, showed no significant differences between treatment and control groups with regard to suicide attempts at post-treatment and between group differences in suicidal ideation were not reported (Rathus \& Miller, 2002). A small quasi-experimental randomized controlled trial of DBT for suicidal adolescent inpatients demonstrated no superior effects to a treatment-as-usual condition, but did demonstrate significant pre to post reductions in suicidal ideation that were maintained at a follow-up evaluation (Katz et al., 2004). This quasi-experimental trial established the feasibility of implementing DBT for adolescents in inpatient settings. 
In a randomized trial of DBT for outpatient adolescents who reported recent and repetitive self-harm in Norway, DBT outperformed enhanced usual care in a 19-week trial (Mehlum et al., 2014). In that study, adolescents in the DBT group reported fewer self-harm incidents across the 19 week study period, reported lower suicidal ideation scores after week 15 of the study period, and displayed lower clinician rated depressive symptoms (but not self-reported depressive symptoms) than adolescents in the enhanced usual care control condition (Mehlum et al., 2014). A German-translation of this same program showed promising results in an open trial (Fleishaker, 2011).

A trial of multisystemic therapy for youths presenting with psychiatric emergencies has also been conducted (Huey et al., 2004). Youths presenting with psychiatric emergencies were randomly assigned to either immediate hospitalization or entrance into multisystemic therapy. At a one year follow-up, multisystemic therapy resulted in significantly fewer youth-reported suicide attempts than did psychiatric hospitalization. However, multisystemic therapy did not show any significant advantage over psychiatric hospitalization with regard to caregiver reported suicide attempts, suicidal ideation, depressive symptoms, or hopelessness.

Donaldson and colleagues (2005) examined a skills-based treatment protocol for reducing suicide-related behaviors. In a trial of the treatment, adolescents reporting to an emergency department or child psychiatric hospital after a suicide attempt were randomly assigned to either the skills-based protocol or a supportive relationship therapy condition. Both treatment groups reported significant decreases in suicidal ideation at three and six month follow-ups, but there were no differences between treatment groups (Donaldson, 
Spirito, \& Esposito-Smythers, 2005). Thus, the skills-based treatment failed to demonstrate any significant advantage over the comparison condition.

Esposito-Smythers and colleagues (2011) conducted a trial of individual cognitive-behavioral therapy for adolescents reporting to a psychiatric inpatient unit with co-occurring suicide-related behaviors and alcohol or marijuana use disorders. In a randomized trial, adolescents were assigned either to the individual cognitive behavioral treatment or a treatment-as-usual control. Treatment consisted of weekly treatment sessions for six months which were then reduced in frequency across the remainder of the year. Adolescents in the treatment group reported significantly fewer suicide attempts at post-treatment. There were no significant reductions in suicidal ideation for either group. Thus, this cognitive-behavioral treatment protocol is promising, but additional research is needed to further demonstrate its efficacy for reducing suicidal ideation and suicide.

Crisis hotlines are also available to adolescents in need of crisis services. Most crisis hotlines are not specific to adolescents and are available by phone or, in some cases, via internet chat features (Substance Abuse and Mental Health Services Administration, 2014), to anyone experiencing suicidal ideation. To date, the effectiveness of crisis hotlines has not been rigorously evaluated, perhaps because of the difficulty of conducting research activities in a crisis hotline setting (e.g., gathering participant assent and parental consent, completing assessments, arranging follow-up assessments). One recent study reported that hotline callers who spoke with counselors trained in the Applied Suicide Intervention Skills Training (ASIST) reported being less depressed, less suicidal, and more hopeful after the call than callers who spoke to counselors not yet trained in the ASIST method (Gould et al., 2013). Though not specific 
to adolescent crisis callers, the ASIST program outlines a three phase plan for suicide hotline representatives to assist suicidal callers. The ASIST model utilizes "connecting," “understanding," and "assisting" phases to guide hotline representatives through exploring the caller's current thoughts and feelings, understanding their reasons for living and dying, and developing a safety plan in a competent and compassionate manner.

Summary of existing prevention programs. While some of the findings reviewed in the preceding sections are promising, few existing prevention approaches have demonstrated significant reductions in suicidal ideation or suicide attempts and none have demonstrated significant reductions in suicide. Also, those few programs with empirical support for reducing suicide-related behaviors (e.g., DBT and individual cognitive behavioral therapy) are in need of independent replication in adolescent samples. The lack of evidence-based programs that produce significant, lasting reductions in suicide-related behaviors among adolescents indicate that additional program development is needed to prevent and treat adolescent suicide-related behaviors.

Notably, the existing approaches to suicide prevention in adolescence fall predominantly within the domain of indicated prevention. Typically, these programs identify adolescents in need of mental health services via hospital inpatient units and emergency departments, often after the adolescent has made a suicide attempt or experienced a severe episode of suicidal ideation (e.g., YST I and YST II, rapid response teams, multisystemic therapy). As a result, these programs are designed to be intensive treatment approaches in order to provide an appropriate level of care for high-risk or acute-risk clients. These approaches to patient identification are located far down the negative trajectory of suicide-related behavior and identify adolescents already 
experiencing clinically significant symptoms. These services address an established pattern of thoughts and behaviors, requiring long-term intervention, and likely result in high therapist demand, extensive demands for documentation and supervision, and a high cost per adolescent treated. These programs are, therefore, resource intensive, potentially limiting the ease with which they may be disseminated to and implemented within a broad range of mental health care settings. That is, where resources are scarce, programs with a high resource demand are not likely to be adopted or, once adopted, to remain sustainable (Glasgow, McKay, Piette, \& Reynolds, 2001). Ideally, program development should not only yield efficacious programs, but should also take into account systematic barriers to dissemination and implementation, including the limited resources available for treatment in many settings.

Identifying adolescents in need of suicide prevention services after an episode of severe suicidal ideation or a suicide attempt fails to address adolescents' experiences of personal distress and the mental health burden associated with suicidal ideation. Identification of adolescents in need of prevention services based on an episode of severe suicidal ideation or a suicide attempt may also fail to identify the estimated nearly $50 \%$ of deaths by suicide that occur on the first suicide attempt (Proulx, Lesage, \& Grunberg, 1997; Rorsman, 1973). Thus, there is specific need for additional universal and selected suicide prevention program development aimed at reducing or preventing the spectrum of suicide-related behaviors prior to adolescents' first suicide attempts or suicidal crises. Development of universal and selected prevention programs coincides with the 2010 Progress Review of the National Strategy for Suicide Prevention, which called for the 
development and evaluation of programs targeting non-clinical suicide risk (Suicide Prevention Resource Center and SPAN USA, 2010).

Developing prevention programs targeting non-clinical levels of suicide risk, however, raises a unique set of feasibility issues. One particular challenge is the limited availability of practitioners and other personnel within the mental health care system. Given that $17.0 \%$ of adolescents reported experiencing suicidal ideation in the previous 12 months (Centers for Disease Control and Prevention, 2014b), programs that target adolescents early in the developmental trajectory of suicide-related behaviors will need to intervene on a broad scale - and thus even a small amount of direct contact from a mental health care professional will add a great deal of strain to an already overburdened system. Suicide prevention programs for adolescents typically have been designed for schools (Isaac et al., 2009; Wyman et al., 2010) and emergency departments (King et al, 2006, King et al., 2009, King, Gipson, \& Horwitz, 2014; Robinson et al., 2009). Use of school settings and emergency department settings may have been done with the goal of not placing additional demands on the time of mental health practitioners by involving nonmental health personnel in the intervention process. However, time for training teachers or hospital staff is likely to be limited and the personnel required to sustain programs may not be feasible in resource-depleted areas. For example, emergency rooms, while often characterized by long wait times, may not have staff available to conduct interventions once programs are no longer supported by research funds. Similarly, teachers and school counselors, already overburdened, may not have the time to personally intervene with such a large number adolescents. Any new program development targeting non-clinical suicide risk will need to take into account the feasibility of the developed treatment for 
dissemination and implementation in community settings (Asarnow \& Miranda, 2014).

For the purpose of broad universal or selected suicide prevention programs, this will require the development of low-cost interventions with relatively little direct contact with a mental health professional. Such programs will also need to be low-cost to allow dissemination to a large pool of individuals.

Table 1. Summary of Adolescent Suicide Prevention Programs

\begin{tabular}{lll}
\hline Citation & Program Title & Implementation Site/Method
\end{tabular}

Universal

Wyman et al., $2010 \quad$ Sources of Strength $\quad$ Schools

Quinnett, 1995 Question, Persuade, Refer Varies

Zenere \& Lazarus, $1997 \quad$ Dade County Public Schools

Schools prevention

initiative

Selected

King, Gipson, \& Horwitz, $\quad$ Teen Options for Change Emergency Department 2014

Gipson et al., $2014 \quad$ Links to Enhancing Emergency Department

Teens' Connectedness

Indicated

King et al., $2006 \quad$ Youth-Nominated Emergency Department

Support Team I

King et al., $2009 \quad$ Youth-Nominated Emergency Department 


\section{Support Team II}

Greenfield et al., 2002

Rapid Response Teams

Dialectical Behavior

Fleishaker, 2011; Katz et

al., 2004; Mehlum et al.,

2014; Rathus \& Miller,

2002;

Donaldson, Spirito, \&

Esposito-Smythers, 2005

Esposito-Smythers et al., 2011

Huey et al., 2004

Gould et al., 2013

Carter et al., 2007
Skills-based treatment

Individual Cognitive

Behavioral Therapy

Multisystemic Therapy

ASIST

The EDge Project
Emergency Department

Mental Health Care Providers
Emergency Department and

Child Psychiatric Unit

Inpatient Psychiatric Unit
Psychiatric Unit

Crisis Hotlines

Mailed Postcards after

Hospitalization

\section{A Way Forward for Developing Suicide Prevention Programs.}

Current suicide prevention efforts, while demonstrating some promise, have not generally proven efficacious. Their reliance on suicide attempts or suicidal crises as means of case identification occurs far down the continuum of suicide-related behaviors and requires the programs to be intensive. It also results in missed cases, where adolescents die by suicide without having made a previous suicide attempt. However, moving the point of intervention toward addressing non-clinical levels of suicide risk will result in a much larger number of adolescents eligible for such mental health services. 
The use of web-based programs offers a low-cost means for delivering mental health services. A web-based delivery format would allow for intervention recipients to complete a program with minimal effort from a mental health profession. More specifically, use of a web-based format would allow for dissemination on a large scale. Furthermore, while web-based prevention efforts may not be suitable as a standalone intervention for acute-risk clients, this format may be appropriate for addressing nonclinical suicide risk in a universal or selected prevention program.

In an effort to increase the sustainability of and accessibility to mental health services, prevention programs using web-based formats have been developed and found to be efficacious (e.g., Calear, Christensen, Mackinnon, Griffiths, \& O'Kearne, 2009; Ybarra \& Eaton, 2005). For example, the MoodGYM intervention is an online cognitivebehavioral universal prevention program for adolescents consisting of five 20-40 minute modules. A large cluster randomized controlled trial of the MoodGYM program in 30 Australian schools reported small-to-medium reductions in anxiety symptoms at posttreatment and six month follow-up evaluations, as well as a small-to-medium significant reduction in depressive symptoms among adolescent boys (Calear et al., 2009).

One smart phone application has been developed to provide links to emergency and mental health resources, called ASK and Prevent Suicide (Mental Health America of Texas, 2013). While this application aims to connect at-risk individuals to potential service providers, it does not directly target suicide-related behaviors. A web-based training program for the Question, Persuade, Refer (QPR) intervention program has also been developed, which allows for the training of "gatekeepers" - individuals trained to recognize the warning signs of suicide and ask basic questions to engage potentially 
suicidal individuals and refer them to treatment. The web-based QPR training program does not provide a direct intervention for suicide prevention, but does bring the realm of suicide prevention training into an online format. Research demonstrates that the online QPR training program can increase participant knowledge and intentions to engage in specific behaviors, comparably to similar in-person programs (Lancaster et al., 2014).

Finally, a 10-week computer-based cognitive behavioral therapy program to address suicide risk, Reframe-IT, has been developed and is being tested for use in school settings (Robinson et al., 2014). The Reframe-IT program utilizes eight cognitivebehavioral modules that include videos for verbal delivery of therapy, diaries, and homework assignments to help students at risk for suicidal behaviors. The modules include agenda setting, emotion recognition, addressing automatic negative thoughts, activity scheduling, problem solving, and cognitive restructuring, among others. The Reframe-IT program utilize a mental health practitioner to set up appointments, review weekly suicide screens, and remain present during the adolescents' use of the program (Robinson et al., 2014). An RCT of the program is currently underway and results are not yet available.

Overall, technology-based (including web-, computer-, and phone-based) programs show potential for addressing mental health issues - and are an emerging front for psychotherapeutic interventions (Bennett \& Glasgow, 2009). The use of electronic interventions has a number of advantages, notably (a) wide availability, wherever users have computer or smart phone access, limiting the need for direct service providers, (b) privacy, to reduce concerns regarding stigma, and (c) high fidelity of intervention delivery. 
The primary goal of this dissertation study is to develop and evaluate a brief, webbased program for the prevention and treatment of suicidal ideation among adolescents at elevated, but not imminent, risk of suicide-related behaviors. A goal of this selective prevention program is to address the mental health burden of suicide-related behaviors upstream, prior to their onset in adolescents. A selective prevention approach may not require the same intensity of services as is required in indicated prevention or intervention approaches after an adolescent has made a suicide attempt or experienced a suicidal crisis. Another goal of this research is to develop a program that requires minimal staff time for training and implementation, while ensuring intervention fidelity, in order to minimize barriers to large-scale dissemination and implementation of the program, should it prove efficacious. 


\section{SUICIDAL IDEATION: FROM A THEORETICAL MODEL TO A NOVEL PREVENTION PROGRAM}

Prior to developing a suicide prevention program targeting non-clinical suicide risk by reducing or preventing suicidal ideation, it is important to consider theoretical conceptualizations of suicide-related behaviors. Theories of suicide-related behaviors propose risk factors for those behaviors. These theoretically-derived risk factors, if substantiated by empirical findings and if they are amenable to change, may then serve as potential targets for intervention.

\section{The Interpersonal-Psychological Theory of Suicide}

Few theories of suicide-related behaviors have been proposed over the past century and fewer still have been empirically evaluated with favorable outcomes. (For a thorough review of theoretical models of suicide-related behaviors, see Barzilay and Apter, 2014.) One theory in particular has received a great deal of empirical evaluation in recent years - the interpersonal-psychological theory of suicide (the IPTS; Joiner, 2005; Van Orden et al., 2010). The IPTS attempts to integrate a large body of empirical literature that identifies risk factors for suicide-related behaviors into a single, parsimonious model. In its simplest form, the IPTS proposes that dying by suicide requires both the ability to end one's own life (called the acquired capability to enact lethal self-injury) and the desire to do so (called the desire for death; Joiner, 2005). The inclusion of the desire for death in the IPTS corresponds directly to the definition of suicide previously outlined: That suicide is a fatal, self-inflicted act (thus requiring an individual be capable of taking his or her own life), with some, non-zero intent to die (at least some desire for death). 
The present research will draw upon the IPTS (Joiner, 2005; Van Orden et al., 2010). Figure 2 depicts the IPTS as a conceptual diagram as it was originally proposed in Joiner in 2005.

Figure 2. The Interpersonal-Psychological Theory of Suicide

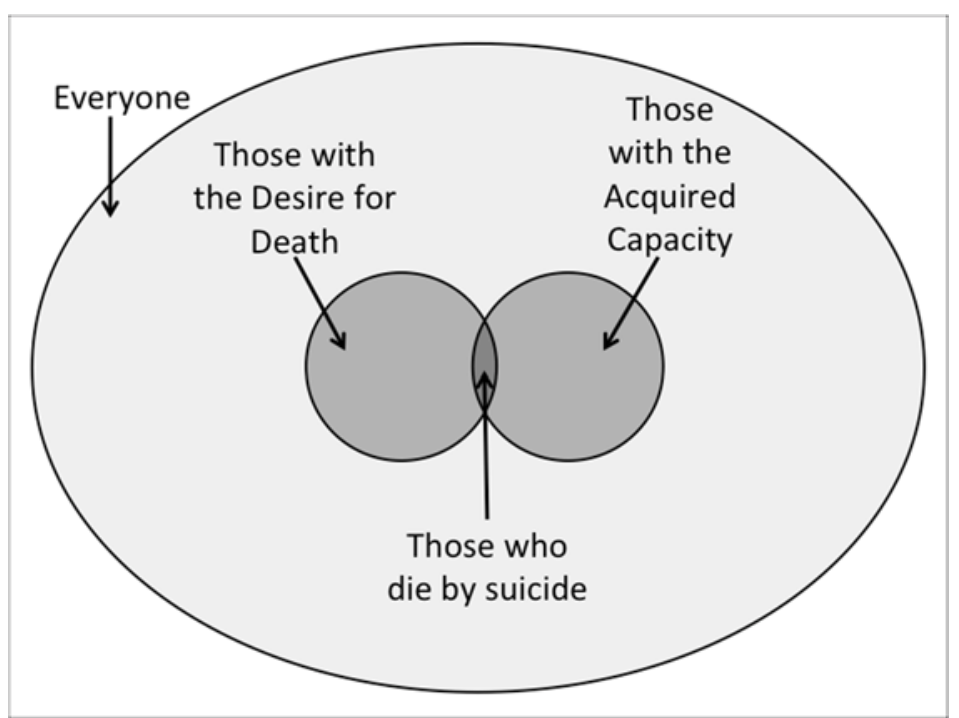

The acquired capability to enact lethal self-injury. The ability to take one's own life, termed the acquired capability to enact lethal self-injury, is viewed as a learned capacity acquired through repeated exposure to painful and provocative events (Joiner, 2005). Broadly conceptualized, painful and provocative events may include experiences that involve actual pain (e.g., broken bones, accidents, self-harm behaviors, repeated abuse) or imagined pain and dangerous situations (e.g., viewing painful events, mental rehearsal of a suicide plan, repeated exposure to danger such as military combat). The acquired capability to enact lethal self-injury is "acquired;" consistent with evolutionary psychological theories, life-threatening situations should be evolutionarily relevant, 
activating a neural fear response (Ohman \& Mineka, 2001), and so individuals must overcome this fear of pain and death. Once that fear response has been sufficiently overcome, an individual is thought to have acquired the capability to end his or her own life. The theory proposes that suicide, and suicide attempts of high lethality, require the acquisition of the acquired capability to enact lethal self-injury. Suicide attempts of low lethality are considered painful events that may contribute to the acquired capability. The acquired capability to enact lethal self-injury is viewed as static in that, once acquired, it is maintained. Therefore, it is not considered amenable to change and so not an efficient target for preventive interventions.

The acquired capability to enact lethal self-injury alone is not sufficient for an individual to die by suicide, as not all those who are able to die by suicide actually do so. Instead, the IPTS proposes that the joint presence of the acquired capability and the desire for death is necessary for suicide; thus, suicide only occurs when an individual has both the capability to take his or her own life and the desire to die.

The desire for death. The desire for death is an individual's subjective desire to cease living. It is roughly equivalent to the definition of suicidal ideation (Van Orden et al., 2008). As such, the desire for death is particularly relevant to the current project, which focuses on the reduction and prevention of suicidal ideation. The desire for death is thought to be modifiable and amenable to change. In the IPTS, the desire for death is comprised of two factors: a sense of perceived burdensomeness (e.g., "My life is a drain on others") and thwarted belongingness (e.g., "There is nobody I can turn to"; Joiner, 2005), which may result from a variety of circumstances. The IPTS proposes that both perceived burdensomeness and thwarted belongingness are necessary for the desire for 
death, such that the joint presence (or interaction) of both factors leads to the strongest desire for death. Since perceived burdensomeness and thwarted belongingness are subjective perceptions of current life states, rather than stable conditions, both may be addressed via preventive interventions.

Thwarted belongingness is comprised primarily of the belief that one's interpersonal relationships are unsatisfactory. The core component of thwarted belongingness is a perception of social isolation or disconnection from others (Joiner, 2005). Thwarted belongingness bears close similarity to a number of risk factors for suicidal ideation among adolescents, including poor family connectedness, a perceived absence of caring adults, and social isolation (e.g., Eisenberg \& Resnick, 2006; Halpert, 2002). Thwarted belongingness has also been operationalized as lack of social support and number of close friendships, and is conceptually congruent with connectedness-based prevention programs. Chronically strained relationships with family, close friends, and romantic partners have been associated with more severe suicidal behaviors in adolescence (e.g., Pettit et al., 2011) and adults (e.g., Chen et al., 2013).

Further, thwarted belongingness is congruent with other psychological theories of well-being, including the "need to belong" proposed by Baumeister and Leary (1995) as a basic human need and motivation. Thwarted belongingness is also conceptually similar to relatedness, one of the three core components of well-being in self-determination theory (Deci \& Ryan, 2000, 2008; Hill \& Pettit, 2012) and with Maslow's (1943) need for belongingness in the hierarchy of human motivations.

In their 2010 review of the literature, Van Orden and colleagues concluded that the association between suicide-related behaviors and thwarted belongingness was 
consistently demonstrated in the extant literature. Further evidence for the association between thwarted belongingness and suicide-related behaviors has been reported in several studies, including samples of young adults (Joiner et al., 2009), older adults (Marty, Segal, Coolidge, \& Klebe, 2012), college students of varying race and ethnicity (Anestis, Bagge, Tull, \& Joiner, 2011; Davidson, Wingate, Grant, Judah, \& Mills, 2011; Davidson, Wingate, Rasmussen, \& Slish, 2009; Freedenthal, Lamis, Osman, Kahlo, \& Gutierrez, 2011; Hill \& Pettit, 2012; Lamis \& Lester, 2012; Rasmussen \& Wingate, 2011; Tucker et al., 2013; Van Orden, et al., 2012; Wong, Koo, Tran, Chiu, \& Mok, 2011), American Indian/Alaskan Native adults (O’Keefe, Wingate, Tucker, RhoadesKerswill, Slish, \& Davidson, 2014), military personnel (Bryan, Cukrowicz, West, \& Morrow, 2010; Bryan, Morrow, Anestis, \& Joiner, 2010), and adolescents (Czyz et al., in press). However, some studies failed to find a significant association between thwarted belongingness and suicidal ideation among adolescents (Merchant, 2010) and military personnel (Bryan, Clemans, \& Hernandez, 2012; Bryan, Ray-Sannerud, Morrow, \& Etienne, 2012). Of note, thwarted belongingness has been a common target of suicide prevention programs (e.g., King et al., 2006, 2009).

The second factor that constitutes the desire for death is perceived burdensomeness. Perceived burdensomeness is comprised primarily of the belief that one's self has become a burden on others (Van Orden et al., 2010) or that one's existence is a drain on the resources of others or on society as a whole (Joiner, 2005). Van Orden and colleagues (2010) also describe perceived burdensomeness as containing an aspect of affectively-laden cognitions of self-hatred, though this facet of perceived burdensomeness has not yet been included in existing measures of perceived 
burdensomeness nor has it been examined in the empirical literature. Perceived burdensomeness is conceptually congruent with Durkheim's (1897) model of altruistic suicide, in which an individual believes that his or her death will benefit others. It is also congruent with traditional Harakiri (or Seppuku) and Suttee suicides, which have historically been "culturally sanctioned" in various cultures and in specific circumstances (Chen, Wu, Yousuf, \& Yip, 2012, p. 134). Perceived burdensomeness also has roots in Sabbath's (1969) theory of suicide in which suicidal adolescents were thought to view themselves as being expendable family members, even when family members disagreed with that notion, highlighting the aspect of burdensomeness as an individual's perception of their current life state.

Most empirical research examining perceived burdensomeness has used the Interpersonal Needs Questionnaire and has demonstrated a consistent significant association between perceived burdensomeness and suicidal ideation (e.g.,; Bryan, 2011; Freedenthal et al., 2011; Hill \& Pettit, 2012, 2013; Marty et al., 2012; Van Orden et al., 2009; Van Orden et al., 2012). Perceived burdensomeness has also been assessed via analysis of suicide notes by coding for burdensomeness-oriented cognitions. Findings using this method have been mixed, though a lack of perceived burdensomeness expressed in suicide notes should not be taken as evidence that perceived burdensomeness was not present (Cox et al., 2011; Gunn, Lester, Haines, \& Williams, 2012; Joiner et al., 2002; Pettit et al., 2002).

In their 2010 review, Van Orden and colleagues concluded that the association between suicide-related behaviors and perceived burdensomeness was consistently demonstrated in the extant literature. Further evidence for the association between 
perceived burdensomeness and suicide-related behaviors has been reported in several studies. Since that time, correlations between perceived burdensomeness and suicidal ideation have been demonstrated among samples of young adults (Joiner et al., 2009), older adults (Jahn \& Cukrowicz, 2011; Jahn, Cukrowicz, Linton, \& Prabhu, 2011; Marty et al., 2012; Van Orden, Cukrowicz, Witte, \& Joiner, 2012), college students of varying race and ethnicity (Anestis et al., 2011; Davidson et al., 2011; Davidson et al., 2009; Freedenthal et al., 2011; Hill \& Pettit, 2012; Lamis \& Lester, 2012; Rasmussen, Slish, Wingate, Davidson, \& Grant, 2012; Rasmussen \& Wingate, 2011; Tucker et al., 2013; Van Orden, et al., 2012; Wong et al., 2011), American Indian/Alaskan Native adults (O’Keefe et al., 2014), military personnel (Bryan, Clemans, \& Hernandez, 2012; Bryan, Cukrowics, et al., 2010; Bryan, Morrow, et al., 2010; Bryan, Ray-Sannerud, et al., 2012), Spanish-speaking adult women (Garza \& Pettit, 2010), and adolescents (Czyz et al., in press; Hill et al., in press; Merchant, 2010). Associations between perceived burdensomeness and a past suicide attempt have also been demonstrated in samples of military personnel (Bryan, Ray-Sannerud, et al., 2012; Monteith, Menefee, Pettit, Leopoulos, \& Vincent, 2013) and clinical outpatients (Anestis \& Joiner, 2011). The association between perceived burdensomeness and suicide has been investigated on two occasions, though different conclusions were reached in each (Cox, et al., 2011; Gunn, et al., 2012). Of note, perceived burdensomeness has not yet been utilized as a target in suicide prevention programs.

\section{The joint presence of thwarted belongingness and perceived}

burdensomeness. The IPTS proposes that the desire for death, or suicidal ideation, occurs in the joint presence of both perceived burdensomeness and thwarted 
belongingness (Joiner, 2005). Of note, Van Orden and colleagues (2010) put forth that perceived burdensomeness or thwarted belongingness alone may predict "passive" or mild suicidal ideation, whereas the joint presence of both perceived burdensomeness and thwarted belongingness (that is, the interaction of these two factors) should predict "active" or severe suicidal ideation. The differentiation is difficult to examine, as the literature rarely distinguishes between active and passive forms of suicidal ideation, nor has an operational definition for such terms been generally agreed upon. Typically, studies of the hypotheses of the IPTS have considered either the associations between suicidal ideation, perceived burdensomeness, and thwarted belongingness individually (i.e., correlations between suicide and thwarted belongingness, correlations between suicide and perceived burdensomeness), as reviewed above, and/or by examining the interaction between perceived burdensomeness and thwarted belongingness.

Of critical importance to the IPTS, however, the hypothesis that the joint presence of thwarted belongingness and perceived burdensomeness will be most strongly associated with severe suicidal ideation has also been tested. In the case of perceived burdensomeness, the IPTS hypothesizes that perceived burdensomeness and thwarted belongingness interact to predict suicidal ideation. The interaction between perceived burdensomeness and thwarted belongingness has been tested in several empirical studies (Anestis \& Joiner, 2011; Bryan, Clemans, et al., 2012; Davidson, Wingate, Slish, \& Rasmussen, 2010; Joiner, et al., 2009; Wong, et al., 2011). The interaction between perceived burdensomeness and the acquired capability to enact lethal self-injury has also been tested (Anestis \& Joiner, 2011; Bryan, Clemans, et al., 2012; Bryan, Morrow, et al., 2010; Joiner, et al., 2009). 
Wong and colleagues (2011) tested the interaction between perceived burdensomeness and thwarted belongingness in a sample of Asian American college students. They reported a significant interaction effect, controlling for the main effects of perceived burdensomeness and for thwarted belongingness. Similarly, Joiner and colleagues (2009) tested for this same interaction effect in a sample of young adults and found that the interaction between perceived burdensomeness (as measured by a mattering scale) and social support (a proxy for thwarted belongingness) significantly predicted suicidal ideation, controlling for six month and lifetime incidence of depression, as well as the main effects of both mattering and social support. In Joiner and colleagues' (2009) study, though, the main effect of mattering on suicidal ideation was not significant.

Others have tested the relation between perceived burdensomeness and the acquired capability to enact lethal self-injury, or between all three components of the IPTS, which are hypothesized by the IPTS to predict serious suicide attempts. Bryan and colleagues (Bryan, Clemans, et al., 2012) tested the interaction between perceived burdensomeness and the acquired capability to enact lethal self-injury in a study of two samples of military personnel - those seeking services for mild traumatic brain injury and those seeking outpatient mental health services. In the former sample, the authors identified a significant interaction, indicating that those with elevated scores on both measures reported significantly higher suicidal ideation scores, controlling for sex, age, depressive symptoms, PTSD symptoms, traumatic brain injury symptoms, and the significant main effects of both burdensomeness and the acquired capability. The authors 
then replicated their analysis in the latter sample and reported similar results in the second sample.

Another study by Bryan and colleagues (2010) tested whether perceived burdensomeness, thwarted belongingness, the acquired capability to enact lethal selfinjury, as well as all of the possible two-way and three-way interactions among them, significantly predicted past suicide-related behaviors. The study by Bryan and colleagues (2010) found that only the acquired capability to enact lethal self-injury, and the interaction between the acquired capability and perceived burdensomeness were significant predictors of suicidal history, after controlling for age, gender, and both positive and negative affect, though these covariates alone accounted for nearly $36 \%$ of the variance. Further tests of the form of the interaction indicated that those high in both perceived burdensomeness and the acquired capability had the highest scores on the measure of suicidal history.

Davidson and colleagues (2010) also tested the interactions between perceived burdensomeness and thwarted belongingness and between perceived burdensomeness and the acquired capability simultaneously, in a sample of 115 African American college students. In the first step, gender, age, marital status, and income did not significantly predict suicidal ideation. In the second step, adding the three IPTS components significantly improved the amount of variation in suicidal ideation accounted for by the model. The third and fourth steps show that the two-way and three-way interactions among the three IPTS constructs significantly predicted suicidal ideation. The report, however, only provided data for each model step, without differentiating the effects of 
the individual variables entered in each step. The significant interaction terms were not analyzed further.

Two studies tested interactions between perceived burdensomeness and other IPTS factors in the prediction of suicide attempts, rather than suicidal ideation: In a second study reported by Joiner and colleagues (2009), described above, the authors tested the three-way interaction between perceived burdensomeness, thwarted belongingness, and past suicide attempts in predicting the presence or absence of a recent suicide attempt. The authors analyzed a sample of 313 young adults referred for treatment for a recent suicide attempts or severe suicidal ideation. Perceived burdensomeness, the interactions between perceived burdensomeness and thwarted belongingness and between perceived burdensomeness and past suicide attempts, did not significantly predict suicidal ideation. The three-way interaction among the three main effects significantly predicted the presence of a recent suicide attempt, controlling for depressive symptoms, past depression diagnoses, hopelessness, demographic factors, and the main effects and two-way interactions, such that risk was greatest when all three risk factors were elevated.

Building upon the framework of the IPTS, Anestis and Joiner (2011) tested whether the four-way interaction between perceived burdensomeness, thwarted belongingness, the acquired capability to enact lethal self-injury, and negative urgency, thee tendency to act in an impulsive manner to reduce feelings of negative affect, predicted lifetime number of suicide attempts among a sample of nearly 500 adults seeking outpatient services at a community mental health center. The authors hypothesized that negative urgency would increment the ability of the IPTS to predict a 
history of suicide attempts. In the final step of the analysis the interaction between perceived burdensomeness and thwarted belongingness, the three-way interaction between perceived burdensomeness, thwarted belongingness, and the acquired capability to enact lethal self-injury, and the four-way interaction between these three factors and negative urgency all significantly contributed to the prediction of the lifetime number of suicide attempts made by individuals, after controlling for participant sex, depressive symptom scores, and all of the remaining main effects, two-way interactions, and threeway interactions. Further analysis of the form of the interaction revealed that lifetime number of suicide attempts was highest in the presence of high levels of all four risk factors.

\section{To intervene with thwarted belongingness, perceived burdensomeness, or}

both? Given the strong empirical evidence of associations between thwarted belongingness and suicidal ideation, between perceived burdensomeness and suicidal ideation, and between the interaction of these two factors and suicidal ideation, interventions that successfully generate improvements in either (or both) of these factors should prove efficacious for reducing or preventing suicidal ideation. Further, given that the IPTS proposes that the interaction between thwarted belongingness and perceived burdensomeness is necessary for actively desiring death, intervening to reduce either of these factors should be sufficient to prevent serious suicide attempts and suicide.

A number of recent studies have suggested that perceived burdensomeness, relative to thwarted belongingness, may be a stronger risk factor for suicidal ideation. Several studies have reported that, when perceived burdensomeness and thwarted belongingness are simultaneously entered as predictors of suicidal ideation, perceived 
burdensomeness remains significantly associated with suicidal ideation, but thwarted belongingness does not (Anestis \& Joiner, 2011; Bryan et al., 2012; Hill \& Pettit, 2012; Lamis \& Lester, 2012; Merchant, 2010; Monteith et al., 2013; O'Keefe et al., 2014; Van Orden et al., 2008; Wong et al., 2001). These studies span a variety of samples and measurement instruments, further supporting the idea that perceived burdensomeness may be the more potent risk factor for suicidal ideation. These studies indicate that it may be at least as important, if not more important, to address perceived burdensomeness in suicide prevention programs, rather than focusing on thwarted belongingness alone.

Unfortunately, existing prevention programs focus only on thwarted belongingness (connectedness, social support, etc.) and omit perceived burdensomeness, or fail to directly address burdensome cognitions specifically. The omission of perceived burdensomeness is likely a result of the relatively recent introduction of the IPTS and the concept of perceived burdensomeness as well as the extensive literature documenting the relationship between social support and suicide-related behaviors that existed prior to the introduction of the IPTS. Initial correlational evidence indicates that the omission of perceived burdensomeness may represent a missed opportunity for prevention and intervention programs to reduce the risk of suicide-related behaviors.

Although existing prevention and intervention programs may address perceived burdensomeness indirectly, it has yet to be leveraged explicitly as focus of preventive interventions. Thus, the present work attempts to leverage perceptions of burdensomeness as a means for reducing or preventing suicidal ideation among adolescents. On the basis of this premise, the LEAP program, described below, was developed. 


\section{The LEAP Program}

The LEAP program is a selected, computer-based prevention program to reduce perceived burdensomeness. The LEAP Program draws on cognitive-behavioral principals to reduce perceived burdensomeness via two, brief, online modules. The two modules are completed approximately one week apart and can be completed online. Each module requires approximately 20-30 minutes to complete. Each module of the LEAP program contains four parts: Learn, Explore, Assess Your Options, and Plan. The program begins with a greeting and introduction, including an explanation of what it means to feel like a burden on others. Table 2 presents a summary of the LEAP modules and phases.

Table 2. Summary of LEAP Modules and Phases

\begin{tabular}{lll}
\hline Phase & Module 1 & Module 2 \\
\hline Learn & Introduction to the LEAP program; & Brief review of \\
& explanation of perceived burdensomeness; & psychoeducational \\
& psychoeducational presentation of Affect- & material presented in \\
& Behavior-Cognition Triangle. & Module 1. \\
Explore & Identification of target relationship and & Identical to Module 1. \\
& situations in which perceptions of & \\
& burdensomeness occur/do not occur. & \\
Assess Your & Generation of activities to reduce & Identical to Module 1. \\
Options & perceived burdensomeness via hypothesis- & \\
\hline
\end{tabular}




\begin{tabular}{ll}
\hline & testing and activity scheduling. \\
Plan & Detailed planning and scheduling of \\
& identified activities to increase perceived \\
& behavioral control and likelihood of \\
& completion of activities.
\end{tabular}

Learn. Following the greeting and introduction, the LEAP program then moves into the first phase, Learn, a psychoeducational phase created on the basis of cognitivebehavioral theory. It begins by introducing the Affect-Behavior-Cognition Triangle, presented in Figure 3 as a thoughts-actions-emotions triangle, which has been used in various formats in cognitive-behavioral treatments for depression (e.g., the Adolescents Coping with Depression Course; Clarke, Lewinsohn, \& Hops, 1990), also known as the Affect-Behavior-Cognition model (Pluzinski \& Qualls, 1986). The Learn phase uses a series of short vignettes geared toward adolescent experiences to explain what thoughts, emotions, and actions are and how they can influence each other. It also introduces adolescents to the concept of manipulating one element of the thoughts-actions-emotions triangle in order to influence the other elements (e.g., to stop your negative thoughts from influencing your emotions, you could engage in a behavior that boosts your emotional state and distracts you from the negative thoughts). The vignettes are coupled with opportunities for the adolescent to demonstrate an understanding of the concepts via multiple choice questions. Correct answers are praised, with the program advancing. Incorrect answers are clarified and asked again, before the program advances. The final vignette ties this concept back to burdensome thoughts, showing adolescents one 
situation in which burdensome thoughts may arise and, in turn, result in further depressed mood. The Learn phase is critical to the subsequent phases as the concepts introduced in this phase form the basis for the active intervention components in the Explore and Assess Your Options phases. The goal of the Learn phase is to introduce adolescents to the concept that thoughts and emotions can be intentionally and proactively modified. With this understanding, adolescents are equipped to then consider modifying their own negative thoughts and emotions in the following phases.

Figure 3. Learn Phase: The Affect-Behavior-Cognition Triangle

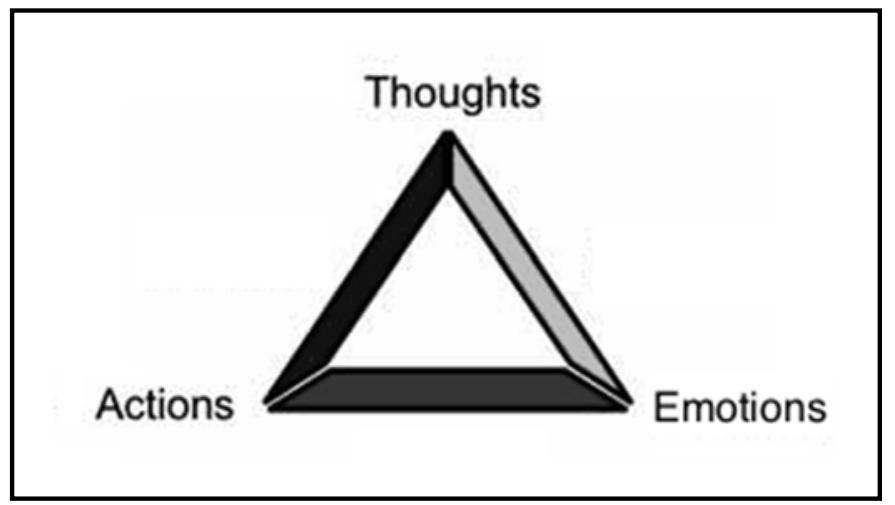

Explore. In the Explore phase, adolescents identify the people, places, and events in which they most commonly have burdensome thoughts. Adolescents first identify all the individuals upon whom they perceive themselves to be a burden. The adolescents are then asked to select a target person, the person upon whom they most often perceive themselves to be a burden or the person that they would most like to no longer feel like a burden on. The adolescent's experience of perceived burdensomeness with this individual then becomes the target of the remainder of the intervention. 
Next adolescents explore times when they have felt like a burden on this target individual. They are encouraged to generate specific examples and describe them - and to rate the severity of these situations with regard to both intensity and frequency, on a 1 to 10 scale. The adolescents also identify times in which they have contributed to the target individual's life or to made that person's life easier or more enjoyable. In this section adolescents are encouraged to think of multiple responses and to be as specific as possible. The process of successfully generating counter-examples, times when the adolescent's relationship with the target individual has not been experienced as burdensome, is intended as a potentially therapeutic exercise. Generating counterexamples is congruent with the cognitive therapy-based approach in which negative cognitions or beliefs are stated and then challenged by means of identifying evidence or counter-examples that contradict the stated cognition or belief (Beck, Liese, \& Najavits, 2005). Finally, responses to these items are stored and appear later in the program, during the Assess Your Options phase, to assist the adolescents in identifying potential activities for reducing their perception of burdensomeness on the target individual.

Assess your options. The third phase of LEAP is Assess Your Options, where adolescents explore ways to reduce burdensome thoughts. Assess Your Options draws on two cognitive-behavioral approaches: challenging distorted cognitions through evidence acquisition (or "reality-checking"; Beck, Rush, Shaw, \& Emery, 1979) and activity scheduling (Clarke, Lewinsohn, \& Hops, 1990). Consistent with previous phases, adolescents are guided through these exercises via a series of short vignettes.

Adolescents begin with an exercise to challenge their distorted cognitions via hypothesis-testing with regard to their burdensome cognitions. Guided by a vignette, 
adolescents are shown an example in which the burdensome cognition is clearly not shared by the target individual. The adolescent is then encouraged to imagine a similar conversation with the target individual, to assess the accuracy of their perception of burdensomeness. That is, because perceived burdensomeness may often be a distorted perception of reality, the process of acquiring evidence that confirms or disconfirms the cognition may be helpful (e.g., "I think I am a burden on my best friend, but my best friend does not think that I am a burden on her."). Adolescents are prompted to explore what they might say and how they could explain their thoughts and emotions to their target individual. The adolescent then has the opportunity to draft how they would initiate such a conversation, including what they might say, when, and where.

Then adolescents begin an exercise that parallels pleasant activity scheduling, a common method of behavioral activation for depression (Cuijpers, van Straten, \& Warmerdam, 2007; Mazzucchelli, Kane, \& Rees, 2009). In this section, adolescents identify activities that will allow them to contribute to the target individual's life in some manner or to share an experience with the target individual that will be positive and nonburdensome (e.g., helping mom by taking care of a younger sibling, planning a pleasant activity, such as a movie night, to spend with the target individual). Adolescents are prompted to consider situations in which they have not perceived themselves to be a burden, and are reminded of the events they reported in the Explore phase. Again through example vignettes, as well as via examples provided by the program and on the basis of the situations they have previously identified as times when they did not perceive themselves to be a burden, adolescents are encouraged to identify activities that allow them to contribute to the life of their target person. Once some possible activities have 
been generated, adolescents are asked to rate the difficulty of completing each activity and the likelihood they will complete each activity. Consistent with the Theory of Planned Behavior (Ajzen, 1991), these ratings are intended to help guide adolescents to select the activity over which they have the greatest perceived behavioral control. Adolescents are encouraged to review their ratings and select the option they think they are both likely to do and that will be easy for them to complete.

Plan. The final phase of the intervention module is the Plan phase, where teens plan when and where to do the activities they have identified. The adolescents are prompted to schedule the two activities they selected in the Assess Your Options phase, one to acquire evidence against burdensome cognitions and another to plan an activity to contribute to the target person in some way. Adolescents identify specific days, times, and places for their selections and plan ways to remind themselves of the planned activities, to maximize the likelihood that they complete the activities. For example, adolescents can elect to program a reminder into their phone or record the event on a calendar or daily planner. The detailed planning of each activity is meant to encourage the adolescent's perceived behavioral control, that is, to increase their perception of being in a position to execute the planned activity. Using careful planning to minimize potential obstacles to completing the planned activities is intended to increase the likelihood that adolescents complete the planned activities.

Module two. The second LEAP module begins with a shortened Learn phase, to serve as a review of the psychoeducational material presented in the first module. Then the Explore, Assess your Options, and Plan phases are repeated, with different vignettes to provide a different set of examples. The purpose of the second module is two-fold: 
First, it provides a larger "dose" of treatment by providing adolescents with a second opportunity to plan and carry out activities to reduce perceptions of burdensomeness on their target individual or to select a different individual for the second module. Second, repetition of the module is intended to help adolescents learn the skills that comprise the LEAP intervention (i.e., hypothesis-testing to challenge distorted cognitions and activity scheduling), so that the adolescents are able to remember and use these skills in the future.

\section{Aims of the Present Studies}

The purpose of the present studies is to conduct Stage I research, as described by Rounsaville, Carroll, and Onken (2001), on the LEAP intervention. Stage I research includes the steps necessary to prepare an intervention for a large-scale randomized controlled efficacy trial. Stage I includes the development of the intervention and manual, feasibility testing, and examination of adherence to the intervention and research protocol. Stage I research may include the use of focus groups, open trials, and smallscale randomized controlled trials. Stage I research allows for (a) collecting data on the acceptability of and participant satisfaction with the intervention, (b) identification of the optimal participant recruitment process and inclusion/exclusion criteria, and (c) examination of the intervention and research design prior to a large-scale study, including identification of flaws in the design, participant adherence to the intervention and research protocols, and feasibility of the protocols. Ideally, Stage I research will also provide some initial evidence to support the potential efficacy of the intervention, or, at minimum, that the intervention does not appear to have iatrogenic effects. Furthermore, successful completion of Stage I research prepares the intervention for Stage II, in which 
large randomized controlled efficacy trials are conducted. Stage III includes

effectiveness trials to examine the portability, generalizability, and cost-effectiveness of the intervention, along with other dissemination and implementation concerns.

The present studies represent Stage I research of the LEAP intervention:

Study 1 . The first study was an open trial of the LEAP program. The open trial was designed to assess the feasibility of the study protocol, the acceptability of the LEAP program to adolescents, and to generate adolescent feedback regarding the LEAP program modules. Specifically, the open trial pursued the following aims:

Aim 1. Examine the feasibility of the study protocol, as evidenced by (a) the ability to identify, screen, and recruit a sufficient sample of adolescents and (b) completion of the baseline and post-intervention assessments by those enrolled in the intervention.

Aim 2. Examine the acceptability of the LEAP program via (a) adolescents' completion of the LEAP program modules, including both the initial and second intervention modules; (b) adolescents' self-reports of satisfaction with the LEAP program and adolescents' feedback regarding the examples, difficulty, and format of the LEAP program; and (c) adolescents' responses to prompts within the LEAP intervention modules.

Aim 3. Examine whether the LEAP program produces significantly lower levels of (a) perceived burdensomeness and (b) suicidal ideation at a one-week followup assessment. Given the sample size of the open trial, results will be considered preliminary. 
Study 2. The purpose of the second research study was to conduct a pilot RCT of the LEAP program. This study built on the open trial with the goal of providing additional evidence for the feasibility and acceptability of the LEAP program, particularly in comparison to a treatment-as-usual control group. It also attempted to provide initial evidence for the efficacy of the LEAP program for reducing cognitions of perceived burdensomeness and suicidal ideation. Specifically, the pilot randomized controlled trial pursued the following aims:

Aim 1. Evaluate participant satisfaction with LEAP and participant use of LEAP modules. Specifically, satisfaction with LEAP modules will be evaluated by examining whether the LEAP program is associated with significantly higher levels of satisfaction ratings as compared to a control condition. Participant use of the LEAP modules will be examined within the LEAP intervention group only. Participant satisfaction, participant use of the LEAP modules, and participant completion of activities planned during the LEAP modules will be evaluated at the post-intervention assessment. Participant satisfaction will also be evaluated six weeks after the intervention.

Aim 2. Examine whether the LEAP program produces significantly lower levels of (a) perceived burdensomeness and (b) suicidal ideation at a post-intervention assessment as compared to a control condition. This aim represents the efficacy test of the proposed project.

Aim 3. Examine whether the LEAP program produces significantly lower levels of (a) perceived burdensomeness and (b) suicidal ideation at a six week follow-up 
assessment as compared to a control condition. This aim represents a test of the maintenance of LEAP program effects six weeks after the intervention. 


\section{STUDY 1: AN OPEN TRIAL OF LEAP}

Study 1 was an open trial of the LEAP intervention. Primary goals of this open trial were to examine the feasibility of the study protocol and examine the acceptability of the LEAP program. The feasibility of the study protocol was assessed via the ability to screen and recruit a sufficient sample of adolescents and by examining participant completion of the baseline and post-intervention assessments. The acceptability of the LEAP program was assessed via adolescents' completion of the LEAP program modules, adolescents' self-reports of satisfaction with the LEAP program, and adolescents' feedback regarding the examples, difficulty, and format of the LEAP program. Finally, a preliminary examination of the efficacy of the program was conducted.

\section{Method}

Participants. Participants were eight adolescents, 13-17 years of age, recruited from a university outpatient mental health clinic and from the surrounding community via distribution of flyers advertising a research study "testing whether a computer program can affect your thoughts and feelings." Inclusion criteria were: 13-17 years of age, endorsement of a perceived burdensomeness score $\geq 10$ on the Interpersonal Needs Questionnaire perceived burdensomeness items, and available Internet access for completion of the intervention modules. Exclusion criteria were: severe suicidal ideation, current psychosocial treatment, and use of psychoactive medications (unless on a stable dose for eight weeks or more).

Procedures. All procedures were reviewed and approved by the Institutional Review Board prior to the start of the study (IRB00008169). Adolescent assent was obtained verbally prior to screening for eligibility via a verbal assent script. Thirty-four 
adolescents completed the initial telephone screening. Eleven satisfied the inclusion/exclusion criteria for participation. The remaining 23 adolescents did not report elevated perceived burdensomeness scores. For three of these 11 adolescents, a parent or guardian could not be reached in order to obtain consent and so they did not participate in the study. For the remaining eight adolescents, a parent or guardian provided verbal consent via a parental verbal consent script (in either English or Spanish) and all eight participated in the study.

Immediately prior to the baseline assessment, the study procedures were reviewed separately with both the adolescent and their parent or guardian. Parental consent and adolescent assent were obtained via online presentation of informed consent documents. Online presentation of informed consent documents was done to provide participants and their parents with an electronic copy of the consent documents, to provide participants and their parents an additional opportunity to ask questions about the study, and to ensure the parent was present during the baseline assessment and available for contact in the event that the adolescent reported a moderate or greater level of suicide risk. Participants then completed the baseline assessment. Of note, recent research supports the use of telephone-based assessment for suicide-related research (Arias et al., 2014). Upon completion of the baseline assessment, participants received remuneration in the amount of $\$ 10$.

Participants were then emailed a link to the first intervention module. One week later a link to the second intervention module was sent via email. Each module was completed via an online survey system, allowing the collection of typed responses to prompts within the intervention modules and verification that adolescents completed the 
modules. One week after the second module was sent (two weeks after the baseline assessment) participants were contacted via telephone to complete a post-intervention assessment. Upon completion of the post-intervention assessment, participants received remuneration in the amount of $\$ 10$. Figure 5 provides an overview of the participant flow and procedures.

Figure 4. Open Trial Participant Flow

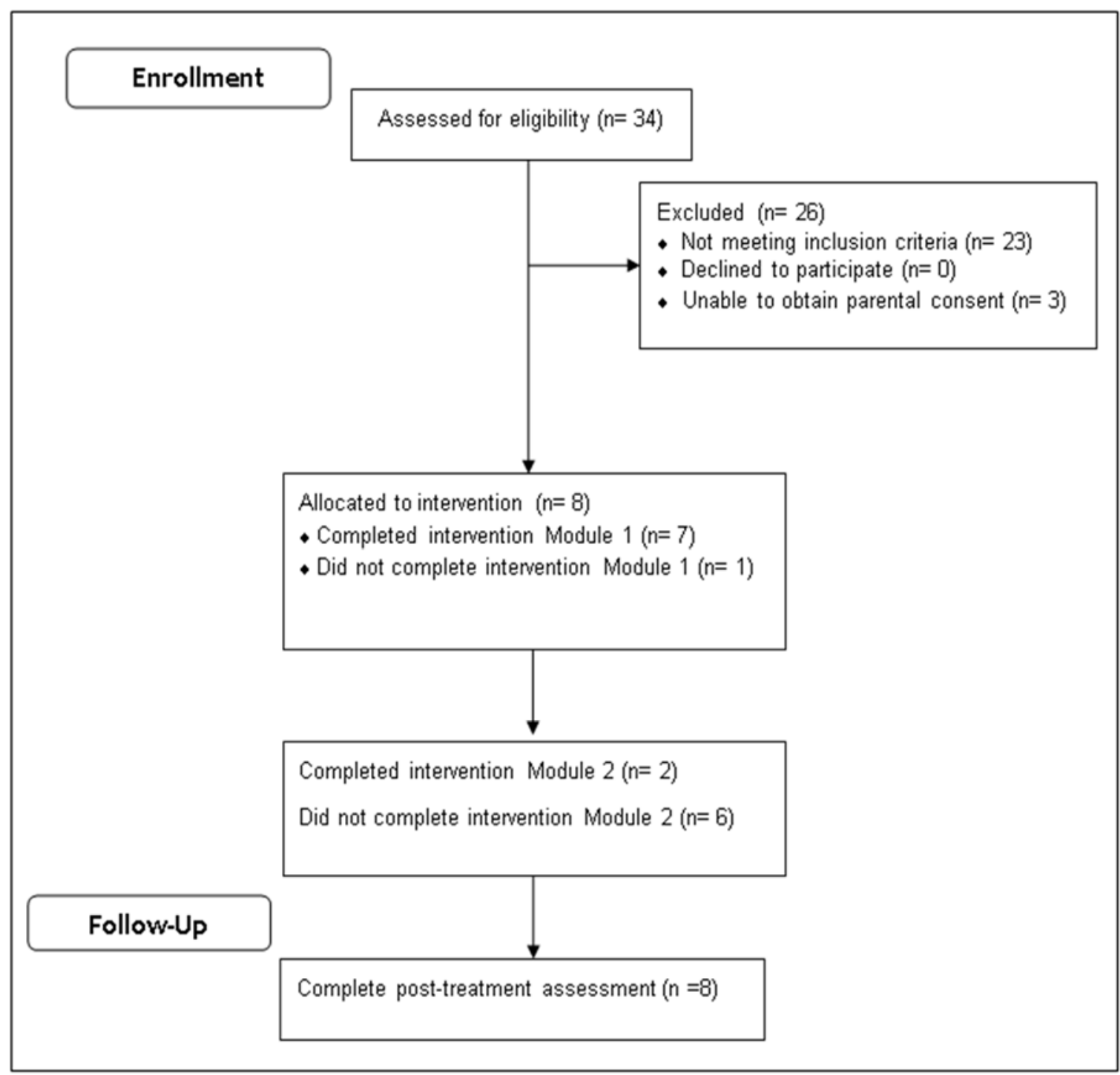


Measures. Measures for Study 1 are described below.

Demographics. Demographic items included age, gender, race, ethnicity, and sexual orientation.

Perceived burdensomeness and thwarted belongingness. The Interpersonal Needs Questionnaire (INQ; Van Orden et al., 2009; Van Orden et al., 2012) is a 15-item self-report questionnaire addressing both perceived burdensomeness (6 items) and thwarted belongingness (9 items). Items are rated on a 7-point Likert scale ranging from "not at all true for me" to "very true for me," with total scores ranging from 6-42 for perceived burdensomeness and 9-63 for thwarted belongingness. Example items on the perceived burdensomeness scale include "These days, the people in my life would be happier without me" and "These days, I think I make things worse for the people in my life." Example items from the thwarted belongingness scale include, "These days, I rarely interact with people who care about me" and "These days, I often feel like an outsider in social gatherings." In our previous work the perceived burdensomeness and thwarted belongingness subscales have consistently shown moderate-to-strong correlations (Hill \& Pettit, 2012, 2013; Hill et al., in press). As such, measurement of thwarted belongingness was included at both pre-intervention and post-intervention assessments in order to examine potential effects of the LEAP program on thwarted belongingness. Internal consistency has been excellent in our previous work with undergraduates (Hill \& Pettit, 2013; Hill et al., in press). The INQ has also demonstrated good internal consistency in an adolescent psychiatric inpatient sample (Hill et al., in press). 
Depressive symptoms. The Reynolds Adolescent Depression Scale -2: Short Form (RADS-2:SF; Reynolds, 1977) was used to assess depressive symptoms. The RADS-2:SF is a 10-item self-report questionnaire designed for use with adolescent populations and is a shortened form of the RADS-2 (Reynolds, 1977). Items are rated on a 1-4 scale, ranging from "almost never" to "most of the time," with total scores ranging from 10-40. Example items include, "I feel happy (reverse scored)" and "I feel I am no good." It has demonstrated excellent reliability and validity in a number of samples (e.g., Horwitz, Hill, \& King, 2011; King, Hill, Wynne, \& Cunningham, 2012). Given the consistent correlation between depressive symptoms and suicidal ideation in our previous work (Hill \& Pettit, 2012, 2013), measurement of depressive symptoms was included to characterize the overall psychopathology of the sample and to examine potential effects of the LEAP intervention on depressive symptoms.

Suicidal ideation. The Beck Scale for Suicide Ideation (BSS; Beck, Steer, Beck, \& Newman, 1993) was used to assess suicidal thoughts occurring in the previous week. The BSS is a 19-item self-report questionnaire that asks participants to select the sentence that best describes their thoughts in the past week from a group of three related sentences. Scores range from 0-2 for each item, with total scores ranging from 0-38. All participants answer the first five questions, assessing general thoughts of suicide. If suicidal thoughts are indicated, participants then respond to 14 additional items. All participants also answer two final items regarding lifetime history of suicide attempts. An example item includes the sentence group, “(0) My reasons for living outweigh my reasons for dying. (1) My reasons for living or dying are about equal. (2) My reasons for dying outweigh my reasons for living." The BSS has demonstrated excellent reliability 
and validity in previous work, including studies of adolescents (Beck et al., 1993; Beck, Kovacs, \& Weissman, 1979; Kumar \& Steer, 1995), and has demonstrated high test-retest reliability across five months among college students (Troister, Davis, Lowndes, \& Holden, 2013). A cutoff score of six or greater has been used to indicate clinically significant suicidal ideation (Lento, Ellis, Hinnant, \& Jobes, 2013). For the present study, a designation of "severe" or greater suicide risk was used as an exclusion criteria, as the LEAP intervention is not intended for those with clinically significant suicidal ideation. A designation of "severe" risk was made for any participant scoring seven or more on select BSS items (including items 10, 12, 14, 15, and 16, which assess reasons for living, current plans, preparations, and expectations of making an attempt, and selfperceived ability to make an attempt). For those with a history of multiple suicide attempts, a designation of "severe" risk was reached with a score of four or more on these select items. No participants in Study 1 received a risk designation of severe or greater.

Satisfaction with the LEAP intervention. The Satisfaction with Services Scale (SSS; Bickman et al., 2010) is a five-item self-report scale designed to evaluate youth satisfaction with an intervention. Items include, "If a friend were in need of similar help, would you recommend our services to him or her?" and "Were the services you received the right approach for helping you?" The first four items are rated on a four point scale ranging from "no, definitely not" to "yes, definitely." Each item is scored 0-3, with total scores ranging from $0-12$. The fifth item provides participants' with an opportunity to provide open-ended feedback. The SSS was developed as part of the Peabody Treatment Progress Battery, which takes a systematic approach to comprehensive measurement of treatment outcomes and satisfaction in youth mental health services (Reimer et al., 2012). 
It has demonstrated a satisfactory degree of internal consistency and evidence of excellent convergent validity (Athay \& Bickman, 2012; Reimer et al., 2012).

Feedback. A feedback form regarding the LEAP program was also included. This form was developed expressly for use with the LEAP program and has not been examined empirically. The feedback form consisted of a series of open-ended questions asking participants for their opinions and feedback regarding the intervention. Key questions included inquiries regarding the number of scheduled activities completed, what participants' liked or disliked about the intervention, and whether the program to difficult or confusing in places. Questions assessing participants' engagement in activities scheduled during the LEAP modules were also included.

Data analysis. As a result of the small sample size and preliminary nature of the study, data analyses consisted primarily of descriptive statistics of the sample, measures, and rates of completion of the LEAP modules. Within-subjects $t$-tests of pre-to-post intervention scores were also conducted, although power was extremely low, even for large effects (i.e., for a large effect size, Cohen's $d$ of $0.8, \alpha=.05$, and $n=8$, achieved power $=0.50$, as computed by the statistical software $\mathrm{G}^{*}$ Power version 3 ; Faul, Erdfelder, Lang, \& Buchner, 2007). As a consequence of the low power associated with the small sample size, analyses with "trend level" significance values of .05-.10 are also reported. In addition, participants' responses to prompts within the intervention and participant feedback form were examined.

\section{Results}

Aim 1a: Feasibility of screening and recruitment. Thirty-four adolescents provided verbal assent and completed the screening for Study 1. Adolescents who 
completed the screening had a mean age of 15.35 years $(S D=1.43$ years $)$ and a mean perceived burdensomeness score of $10.38(S D=6.30)$. Of those who completed the screening, all were 13-17 years of age, all reported having available access to the Internet, and $11(32.35 \%)$ reported a perceived burdensomeness score $\geq 10$. In total, 11 $(32.35 \%)$ met inclusion criteria for entry into the study. Of those who met inclusion criteria, none were deemed ineligible on account of the exclusion criteria (severe suicide risk, current engagement in treatment, or use of psychoactive medications). Of those who were eligible $(n=11)$, parental consent was obtained for $8(72.72 \%)$. For the remaining three, the research team was unable to contact parents in order to obtain parental consent. No parents who were contacted failed to provide consent for their children to participate.

Aim 1b: Feasibility of baseline and post-intervention assessments. Of the 8 adolescents for whom parental consent was obtained, all 8 completed the baseline assessment. The mean age of this final sample was 15.00 years $(S D=1.21$ years $)$. The sample was predominantly female, $(87.5 \%, n=7)$, and $75 \%(n=6)$ reported their ethnicity as Hispanic. With regard to race, $62.5 \%(n=5)$ reporting being White, $12.5 \%$ $(n=1)$ reported being both White and Black, and 25\% $(n=2)$ reported an "other" race. Of the 8 participants who completed the baseline assessment, all 8 completed the postintervention assessment.

Aim 2a: Completion of the LEAP modules. Of the 8 participants who completed the baseline assessment, 7 (87.5\%) completed the first intervention module. Only 2 participants completed the second intervention module, which was sent to participants via email, one week post-baseline. At the post-intervention assessments, 
participants reported completing between 0 and 5 activities, with 7 completing at least one activity.

Aim 2b: Satisfaction with the LEAP program. All participants reported a high level of satisfaction with the intervention on the Satisfaction with Services Scale (Mean = $10.25, S D=1.39$ ). Participants were also asked a series of open-ended questions to provide feedback regarding participants' experiences with the LEAP intervention modules. Participants were asked if any portion of the LEAP intervention modules were too easy, too difficult, or didn't make sense. Two participants reported that portions were too easy, stating only that the program was too simple overall. No participants reported that portions of the intervention were too difficult or didn't make sense. Finally, 7 participants reported that they would use the LEAP program again and 7 participants thought that they were capable of using the techniques they learned in the LEAP intervention modules on their own.

Aim 2c: Examination of participants' responses to prompts within the LEAP intervention modules. Participants' responses to prompts in the intervention module were then examined for the seven participants who completed the first module. Responses were examined to determine if participants understood the prompts in the manner intended by the research team, were replying with the appropriate level of specificity and detail, and to determine if participants were completing the modules.

Learn. In the Learn phase, following presentation of the affect-behavior-cognition triangle, participants respond to prompts asking them to demonstrate knowledge about the relationship between thoughts, emotions, and actions. All participants correctly 
responded to both items, indicating accurate knowledge of the psychoeducational material.

Explore. The first portion of the Explore phase asks participants to identify (a) whom they have felt like a burden on (selecting all that apply) and (b) the person they feel like a burden on the most. For the first item, participants were free to identify as many individuals as they wished, from a list of 15 pre-identified prompts (including an option for "someone else" and specifying a response). One participant selected mother and best-friend; a second participant selected mother, father, and friend; a third participant selected mother, father, grandmother, brother, sister, best-friend, friend, and teacher; a fourth selected best-friend, friend, and boyfriend; a fifth selected mother, father, sister, best-friend, and friend. The two remaining participants selected the "someone else" option; both subsequently indicated not having felt like a burden on anyone.

Regarding the individual whom the participants felt most like a burden on, or whom they would most like to not feel like a burden on, four participants selected mother, one participant identified a friend, and one selected "someone else" and indicated that this person was an aunt. The intervention then focused on reducing perceptions of burden on this identified individual.

In the next portion of the Explore phase, participants were asked to describe times when they have felt like a burden on the target individual as well as times they have contributed to their target individual's life. Responses ranged from general occurrences to specific events: One participant wrote of her mother, "I feel like I ask too much from her. For example, I feel like I ask for food or money too many times, and this annoys 
her." Financial concerns were a common theme; another participant wrote about a tendency to forget and lose things, writing, "Since we are in a financially stressful situation, paying for the things I have lost aren't exactly in the budget and get my mom really upset." Another wrote about "unintentionally influencing my brother to move away from religion." Another elected not to answer this prompt.

Examples of ways participants have contributed to the target individual's life were also varied. One participant described helping her mother, "I've often ran errands for her, like buying groceries, I do some chores around the house to help her out.” Another wrote, "I've showed her new music she likes, I've made her laugh by doing funny things with my dog." Still another noted "Helping my aunt with moving and taking care of my cousin" as helpful activities. One participant opted not to respond and another noted "I don't have any examples." Others responses were more generic, including "I always get good grades" and "I have tried to be the best person I can to my community."

Assess your options. The third phase of LEAP prompts participants to identify ways to reduce burdensome cognitions directed toward the target individual. First, participants are prompted to imagine and script a conversation with the target individual. One participant wrote, "I would say, 'Mom, there is something I'd like to talk to you about. When I don't clean up after myself and close myself off because I'm irritable, I feel as though I'm a burden to you. When you consistently tell me that I never think of others and that I'm as cold as ice, that hurts my feeling and only makes me feel more like a burden to you." Another wrote, "Mom, I feel like you may get annoyed by me sometimes, but I feel like you never put yourself in my shoes. I'm constantly stressed out 
for one reason or another, and often times I feel like I just want to go out and have fun with my friends. I understand that we never have money for doing the "fun" things anymore, but I just wish I had more free time to do whatever I want. I know that you and I can solve this by spending time with each other." The remaining participants wrote down general comments about the conversation they might have.

Participants were then prompted to generate ideas for contributing to the target individual's life, ways that they could help the target individual, or ways that they could contribute to a goal that the target individual has. In order to help the adolescents generate ideas, participants were shown their responses from the Explore phase, regarding ways that they have contributed to this person's life in the past. Responses included, "Give her more free time... be more independent," "setting reminders on my phone to make sure I've cleaned up," "doing the dishes," and "to have a reminder list to write down the things I need to do."

Finally, participants were asked to rate each of their ideas on 5-point Likert-type scales with regard to how easy/difficult each activity is to do (from "very difficult" to "very easy") and how likely/unlikely it is that they would complete each activity (from "extremely unlikely" to "extremely likely"). Then participants were prompted to select one idea to plan - one that they are both likely to do and that is not too difficult. In all cases, participants selected an idea that was ranked as being "easy" or "very easy" to do and "likely" or "very likely" to be completed.

Plan. In the Plan phase participants were reminded of the two actions they were being asked to complete (talk to the target individual and whatever action they selected from their list of ideas). Participants identified the day/date, time, and place for each 
activity and at least one method for giving themselves a reminder. All participants responded to each prompt, planning the time and place for the activity and selected at least one method of reminding themselves. The most commonly selected methods were putting a reminder in their phone and writing the activity down in their planner/calendar. Several participants opted for multiple reminders. Only one participant selected to try and remember to do the activities without a reminder.

Aim 3: Impact of intervention. To assess the potential impact of the LEAP intervention, paired samples t-tests were conducted comparing participants' scores on each study measure at baseline with their post-intervention assessment. Means and standard deviations for the final sample at baseline and for the post-intervention assessment are presented in Table 4. Comparison of perceived burdensomeness at baseline to perceived burdensomeness at post-intervention assessment was statistically non-significant, $t(7)=-1.59, p=.16$.

Baseline and post-intervention comparison of suicidal ideation was also examined. Only four participants reported any suicidal ideation at baseline and all showed reductions in suicidal ideation at post-intervention. A paired samples t-test of suicidal ideation scores at baseline and post-intervention, while statistically nonsignificant, did reveal a trend toward improvement, $t(7)=2.05, p=.08$. Finally, given the associations between perceived burdensomeness and thwarted belongingness and between depressive symptoms and suicidal ideation, paired-samples $t$-tests of thwarted belongingness and depressive symptom scores were also examined, but were statistically non-significant (thwarted belongingness: $\mathrm{t}(7)=-0.79, p=.46$; depressive symptoms: $\mathrm{t}(7)$ $=0.87, p=.41)$. 
Table 3. Means and Standard Deviations of Open Trial Study Measures.

\begin{tabular}{lcc}
\hline Measure & $\begin{array}{c}\text { Baseline assessment } \\
\text { Mean }(S D)\end{array}$ & $\begin{array}{c}\text { 1-Week post-intervention } \\
\text { assessment Mean (SD) }\end{array}$ \\
\hline Perceived burdensomeness & 11.75 & 13.13 \\
& $(6.27)$ & $(6.15)$ \\
Thwarted belongingness & 25.38 & 26.75 \\
& $(11.55)$ & $(11.11)$ \\
Depressive symptoms & 20.87 & 19.38 \\
& $(4.09)$ & $(8.16)$ \\
Suicidal ideation & 2.13 & 0.25 \\
Treatment satisfaction & $(3.18)$ & $(0.71)$ \\
& -- & 10.25 \\
\hline
\end{tabular}

\section{Discussion}

Study 1 was an open trial of a new, web-based brief intervention called LEAP, which was designed to reduce perceptions of burdensomeness among adolescents who reported an elevated level of burdensome cognitions. The purpose of the study was to assess the feasibility of the study protocol, the acceptability of the LEAP program to adolescents, and to generate feedback from the adolescent participants in order to inform refinements to the LEAP modules. An additional aim was to explore the potential impact of the LEAP program for reducing perceived burdensomeness and suicidal ideation. 
Aim 1: Feasibility of the study protocol. With regard to the feasibility of the study protocol, two aspects of the open trial were examined: The ability to recruit a sufficient sample of adolescent participants and completion of the study assessments by those enrolled in the study. The initial goal was to recruit a sample of eight adolescents, which was successfully accomplished. There were no cases in which parents were contacted but declined to allow their adolescent to participate. Of the eight participants who enrolled in the open trial, all eight completed both the baseline and the postintervention assessment, indicating the feasibility of implementing the LEAP study protocol.

Aim 2: Acceptability of the LEAP program. With regard to the acceptability of the LEAP program, findings were mixed. Seven of the eight adolescents completed the first LEAP module. In all cases, the module was completed immediately following the baseline assessment. In contrast, only two of the eight participants completed the second module one week later. It is not clear why the majority of the participants did not complete the second intervention module. It may be that adolescents felt the first module was not useful and so did not choose to complete the second module. This seems like a likely explanation for the two individuals who did not identify any specific individuals upon whom they felt like a burden. Another possibility is that adolescents did not check their email containing the link to the second module, forgot to complete the second module, or did not perceive themselves to be a burden at the time they received the second module. A reminder to complete the second module via text message might result in a higher rate of intervention completion, and so a reminder message was added to the study protocol. 
Participants' self-reported satisfaction with the LEAP program was quite high, indicating that the participants felt that they would use the module again and would recommend it to others. Adolescents' feedback also indicated no substantial problems with the module, as adolescents did not report difficulty with the reading level or confusion regarding specific parts of the intervention modules. Adolescents also reported completing their planned activities, which further supports the acceptability of the LEAP program.

Modifications to the LEAP modules. Based on participants' responses to prompts in the LEAP intervention modules, a number of minor modifications were made to the intervention modules:

In the Learn phase, participants accurately responded to questions evaluating their knowledge of the affect-behavior-cognitions triangle. Thus, it appeared that this phase met its objective for teaching the psycheducational material and so the Learn phase was not modified prior to Study 2 .

For the Explore phase, two participants indicated not having felt like a burden on anyone. It is possible that these individuals had occasional, vague perceptions of being a burden, but did not have burdensome thoughts frequently enough to identify a common target of those thoughts. These responses may indicate the threshold for inclusion in the study was set too low. The inclusion criteria for perceived burdensomeness was increased to help ensure participants are experiencing sufficiently elevated perceived burdensomeness to benefit from the LEAP intervention. The inclusion criterion was raised to a score of $\geq 17$ on the Interpersonal Needs Questionnaire perceived 
belongingness scale, which was one standard deviation above the mean in this open trial. In this way, approximately $15 \%$ of adolescents will meet this inclusion criterion.

In addition, when generating examples of experiences that made the adolescents think they were a burden, several adolescents gave generic examples. The use of generic examples indicates that some participants may need assistance in identifying specific actions that have contributed to the target individual's life. Specificity is critical in this section, as participants' responses return as prompts to help in generating activities to schedule - and non-specific examples may be more difficult for adolescents to plan and execute. As a result, the text of the intervention was modified to provide additional, specific examples, with the intent that a broad range of highly specific examples would guide participants toward identifying specific behaviors. In addition, the instructions were modified to encourage participants to provide specific examples and to use the bank of examples for assistance, if needed.

In the Assess Your Options phase, when asked to plan a conversation with their target individual, several participants wrote down general comments about the conversation they might have rather than scripting how they might begin that conversation. As a result, the instructions were reworded to encourage participants to script an actual opening to this conversation. This was intended to allow participants to practice what they would want to say and to clarify their thoughts.

Adolescents also rated how likely they were to complete each identified activity and how easy each activity would be to complete. This rating and selection step was included to ensure that participants selected activities that could reasonably be completed and to encourage selection of activities over which participants had high perceived 
behavioral control. As all of the adolescents selected appropriate activities, based on their own ratings of which activities were "likely" to be completed and "easy" to complete, no modifications were made to this portion of the Assess Your Options phase.

Given that adolescents completed the majority of the prompts within the Plan phase, no modifications were made to this phase. A summary of each of the modifications discussed above is provided in Table 5 .

Table 4. Summary of Modifications to the LEAP Modules

\begin{tabular}{lll}
\hline Phase & Issue & Modification \\
\hline Learn & -- & -- \\
Explore & Participants did not identify & Inclusion criteria raised to INQ $\geq 17 ;$ \\
& specific target of burdensome & added additional specific examples to \\
& cognitions; difficulty & item prompt \\
& generating examples of & \\
& activities that contribute to & \\
& target individual's life & \\
Assess Your & Participants did not script a & Clarified instructions to encourage \\
Options & conversation & scripting the opening to a \\
& & conversation and practicing \\
Plan & - & - \\
\hline
\end{tabular}

Aim 3: Impact of intervention. Given the small sample size of this open trial, only preliminary analyses of the impact of the LEAP program were possible. While there 
was no evidence in support of an impact on perceived burdensomeness scores at the postintervention assessment, there was a trend toward a reduction in suicidal ideation scores. Without the inclusion of a control group it is not possible to determine if this trend is due to participation in the LEAP modules or whether such reductions were the result of the passage of time.

Strengths and limitations. A strength of this study was its systematic assessment of the feasibility and acceptability of the research protocol and LEAP modules prior to conducting a pilot randomized controlled trial of LEAP. Limitations include the small sample size, an aspect of the open trial design, and restricted racial and ethnic diversity of the sample (which was predominantly Hispanic). In addition, this open trial did not include a follow-up assessment and so was unable to ascertain the feasibility of retaining a high percentage of participants at a follow-up assessment several weeks following the post-intervention assessment. This limitation will be addressed in the pilot randomized controlled trial, Study 2, via the inclusion of a six-week postintervention follow-up assessment.

Conclusions. This study was an open trial of a brief, web-based suicide prevention program for adolescents targeting the adolescents' perceptions of burdensomeness on others. Eight participants enrolled in the intervention, of 11 who met eligibility criteria during an online screen. Of those eight adolescents, all eight completed the baseline and post-intervention assessments, indicating strong feasibility of the study protocol for recruiting and retaining adolescent participants. Of the eight participants, seven completed the primary intervention module, but only two completed the second intervention module, indicating room for improvement in the acceptability of 
the intervention. A number of modifications were made to the intervention modules based on feedback provided by the participants and examination of participants' responses within the intervention modules. 


\section{STUDY 2: A PILOT RANDOMIZED CONTROLLED TRIAL OF LEAP}

The aim of the present study was to conduct a pilot RCT of LEAP, a brief selected prevention program to reduce the level of perceived burdensomeness among adolescents at risk for suicidal thoughts due to cognitions of perceived burdensomeness. Primary goals of this pilot RCT were to (a) evaluate participant satisfaction with the LEAP intervention and participant use of LEAP modules, (b) examine whether the LEAP intervention produces significantly lower levels of perceived burdensomeness and suicidal ideation at one week post-baseline assessment as compared to a treatment-asusual control group, and (c) examine whether the LEAP intervention produces significantly lower levels of perceived burdensomeness and suicidal ideation at a sixweek follow-up assessment as compared to a treatment-as-usual control group. Potential effects of the LEAP intervention on levels of adolescent-reported depressive symptoms and thwarted belongingness at post-intervention and six-week follow-up were also examined. These goals represent tests of the acceptability and initial efficacy of the LEAP intervention, and short-term maintenance of the effects of LEAP, respectively.

\section{Method.}

Participants. Participants were 80 adolescents, 13-19 years of age, recruited from the community via distribution of flyers advertising a research study "testing whether a computer program can affect your thoughts and feelings." In order to facilitate recruitment, the inclusion criteria for age was expanded from 13-17 years of age in the open trial to 13-19 years of age in the pilot RCT. This expanded age range is consistent with other adolescent-focused interventions (e.g., King, Gipson, \& Horwitz, 2014) and with the period across which suicide rates rise in adolescence (Centers for Disease 
Control and Prevention, 2014a). Flyers were distributed at schools, universities, and public gathering places frequented by adolescents. Inclusion criteria were: 13-19 years of age, endorsing a perceived burdensomeness score $\geq 17$ on the Interpersonal Needs Questionnaire perceived burdensomeness items, and having available Internet access for completion of the intervention modules. Exclusion criteria included severe suicidal ideation, current psychosocial treatment, or use of psychoactive medications (unless on a stable dose for eight weeks or more). Additionally, study enrollment was limited to 50 adolescents aged 13-17 years and 30 adolescents aged 18-19 years.

A total of 708 adolescents provided verbal consent and completed the telephone screen, with a mean age of 16.67 years (SD 1.70 years) and a mean INQ perceived burdensomeness score of 11.11 (SD 6.25). Of the 608 adolescents who did not meet the inclusion/exclusion criteria, 593 did not report a perceived burdensomeness score $\geq 17$ on the Interpersonal Needs Questionnaire. The remaining 15 adolescents excluded at the screen reported elevated perceived burdensomeness scores but were receiving psychosocial treatment or psychoactive medication.

Of those who completed the screen, $100(14.1 \%)$ met the screening inclusion and exclusion criteria, 68 of whom were aged 13-17 years and 32 were aged 18-19 years. Of the 32 adolescents aged 18-19 years who met inclusion criteria, 2 declined further participation prior to completing the baseline assessment, for a total enrollment of $n=30$ participants ages 18-19 years. Of the 68 adolescents aged 13-17 years who met inclusion criteria, parental consent was obtained for 53 (77.9\%). Reasons parental consent was not obtained included: being unable to reach a parent via telephone $(n=11)$, parent did not speak English or Spanish $(n=2)$, and parent did not wish their child to participate $(n=$ 
2). Of the 53 for whom parental consent was obtained, 3 were later excluded due to reporting severe suicidal ideation during the baseline assessment, for a total enrollment of $n=50$ participants ages 13-17 years. For those adolescents with severe suicidal idetaion, parents were informed of the adolescents' suicidal thoughts and behaviors and were encouraged to seek treatment immediately. Referral information was provided and further recommendations were made as appropriate. Suicide risk assessment procedures were identical to those reported in Study 1. The total final enrollment was $n=80$.

The 80 participants enrolled in the study reported a mean age of 16.93 years (SD 1.66 years). Participants were predominantly female (68.8\%) and Hispanic (65.8\%). Participants reported their race as follows: white or Caucasian (68.4\%), black of African American (16.5\%), Asian (7.6\%), Native Hawaiian or Pacific Islander (0.0\%), American Indian or Alaskan Native (1.3\%), and other (8.8\%). The majority were born in the United States (70.0\%), were eligible for free lunch at school (56.3\%), and identified their sexual orientation as heterosexual (88.8\%).

Procedures. All procedures were reviewed and approved by the appropriate Institutional Review Board prior to the start of the study. Procedures for acquiring adolescent and parental assent/consent were similar to those in Study 1: Parental consent and adolescent assent were obtained both verbally and via online presentation of informed consent documents prior to the baseline assessment. For 18-19 year old participants, both verbal and online consent documents were completed prior to the baseline assessment, but parental consent was not required. Participants received remuneration in the amount of $\$ 30$ for completing the baseline assessment, $\$ 20$ for 
completing the post-intervention assessment, and $\$ 20$ for completing the follow-up assessment.

After consent/assent was obtained, adolescents completed a baseline interview via telephone and were then randomly assigned to either the intervention condition or control condition. The interviewer assigned the adolescent by opening a sealed envelope into which a card indicating the random assignment had been placed prior to the study, based on the results of random number generator sequence. A stratified random sampling procedure was used to ensure even distribution of adolescents into the LEAP intervention and treatment-as-usual control groups, such that 50 adolescents between 13 and 17 years of age were randomly assigned separately from 30 adolescents $18-19$ years of age. The control condition was an enhanced treatment-as-usual control. Participants in the control condition were emailed information about suicide risk factors and provided with a list of local and national resources for mental health treatment and suicide/crisis counseling. After the follow-up assessment, participants in the control condition were offered the opportunity to participate in the LEAP program and were emailed a link the program. The intervention condition consisted of the LEAP program modules, via links emailed to participants, as well as the materials provided to the enhanced treatment-as-usual control condition.

After completing the baseline assessment, participants were emailed a link to the first intervention module. The module was completed via an online survey system, allowing the collection of typed responses and verification that adolescents complete the module. One week later a link to the second intervention module was emailed to participants. In addition to this, participants in the intervention group were sent up to 
three text reminders to complete the second module. One week after the second module was sent (two weeks after the baseline assessment) participants were contacted via telephone to complete a post-intervention assessment. Six weeks after the postintervention assessment, participants were contacted to complete a follow-up assessment.

Figure 6 provides an overview of the participant flow and procedures. As shown in the Figure, 708 adolescents were assessed for study eligibility, of which 628 were excluded due to a lack of elevated perceived burdensomeness or to current treatment utilization. The remaining 80 completed a baseline assessment and then were randomly assigned at a 1:1 ratio to either the LEAP intervention or treatment-as-usual control condition, resulting in two groups of 40 individuals. As noted, one individual crossed over from the control to the intervention group due to an interviewer error. Two weeks after the baseline assessment, a post-intervention assessment was conducted with 71 adolescents. The remaining 9 adolescents were not able to be reached for participation ( $n$ $=8$ ) or did not wish to participate further in the study $(n=1)$. Six weeks later a followup evaluation was conducted with 69 adolescents. The remaining 11 adolescents were not able to be reached for participation $(n=10)$ or did not wish to participate further in the study $(n=1)$. The final analysis was conducted utilizing the available data from all 80 participants.

Measures. Measures for the pilot RCT were similar to those used in the open trial and included demographic items, the Interpersonal Needs Questionnaire, the Reynolds Adolescent Depression Scale -2: Short Form, the Beck Scale for Suicide Ideation, the Satisfaction with Services Scale, and the LEAP feedback form. Internal 
Figure 5. Pilot Randomized Controlled Trial Participant Flow

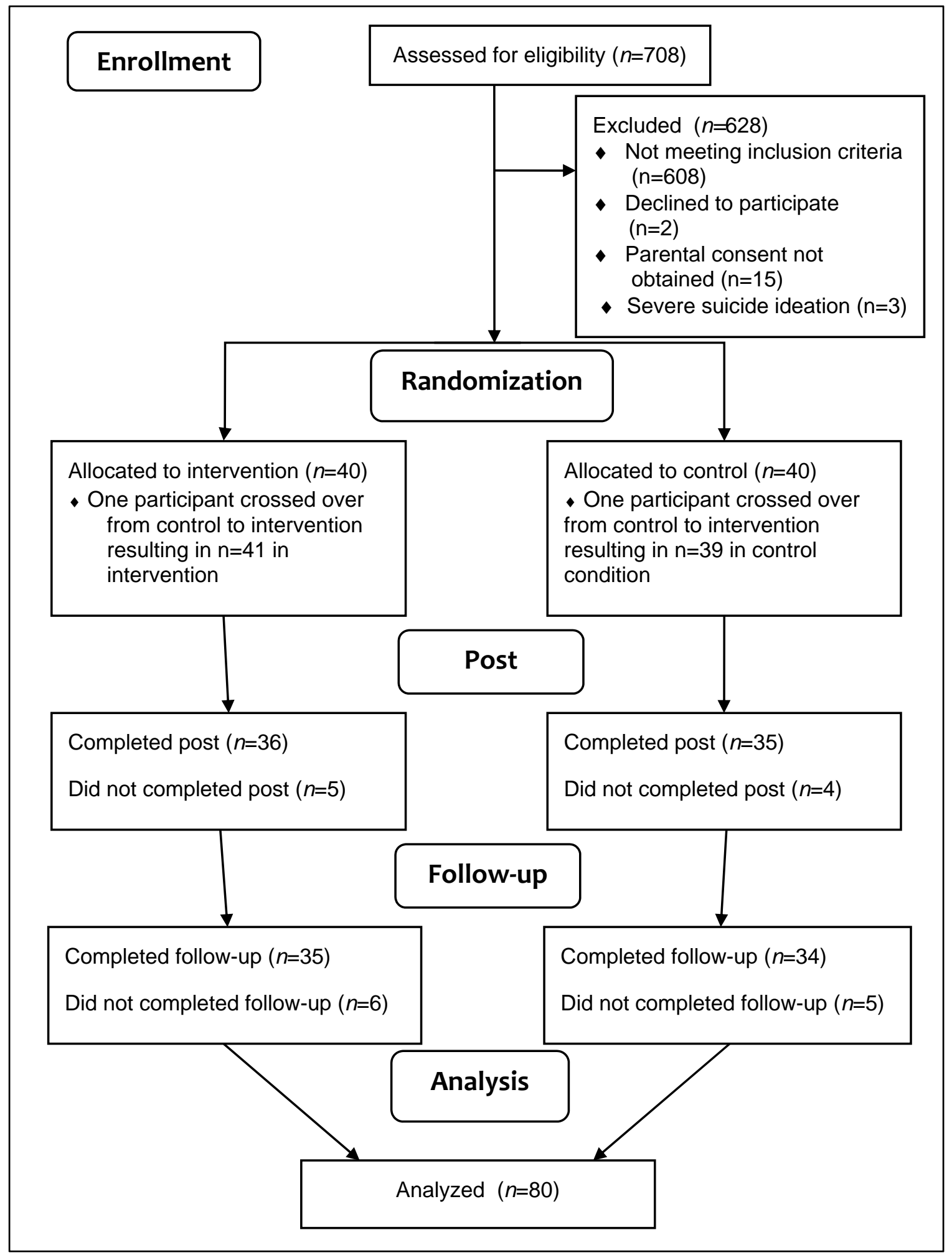


consistencies for each measure were acceptable; Cronbach's alphas for each scale are presented in Table 5 .

Table 5. Internal Consistencies of Measures for Study 2

\begin{tabular}{lccc}
\hline Measure & Baseline & Post- & Follow-up \\
& assessment & intervention & assessment \\
& & assessment & \\
& & .86 & .88 \\
\hline INQ - Perceived Burdensomeness & .80 & .78 & .82 \\
INQ - Thwarted belongingness & .68 & .84 & .80 \\
RADS-2:SF & .70 & .79 & .73 \\
BSS & .64 & .77 & .85 \\
SSS & -- & & \\
\hline
\end{tabular}

Data Analysis. Missing data due to incomplete post-intervention and follow-up assessments occurred at a low rate, with $88.75 \%$ of participants $(n=71)$ completing the post-intervention assessment and $86.25 \%$ of participants $(n=69)$ completing the followup assessment. Missing data were assessed by computing a dummy variable representing the presence or absence of missing data at each time point. Associations between these dummy variables and baseline demographic and clinical characteristics were examined via independent samples t-tests and chi-squared analyses. No significant associations were observed, indicating no evidence of bias due to missing data. Missing data were thus assumed to be missing at random (MAR). Given this assumption, and the low rate of missing data overall, an expectation maximization (EM) algorithm was used to account for missing data. In a simulation study, Newman (2003) reported that, for three- 
wave longitudinal studies where data are MAR or MCAR and 25\% of data are missing, EM, full information maximum likelihood, and multiple imputation perform similarly with respect to introducing minimal bias into statistical estimates.

The data were evaluated for multivariate statistical outliers by examining indices of leverage, influence, and discrepancy. For each analysis, individuals with leverage indices greater than $3(\mathrm{k}+1) / \mathrm{n}$ (where $\mathrm{k}$ is the number of predictors in the model), externally studentized residuals greater than an absolute value of 3.29, or Cook's D values greater than an absolute value of 1 were considered potential statistical outliers (Cohen, Cohen, West, \& Aiken, 2003; Tabachnick \& Fidell, 2013). No individual analysis yielded more than two potential outliers and each analysis was conducted both with and without the outliers. As identical conclusions were drawn from analyses with and without the outliers, data are presented here with the outliers included to better represent the population of interest.

For the evaluation of baseline sample characteristics, Pearson's correlations and independent samples $t$-tests were conducted. For examination of intervention effects, ordinary least squares analyses of covariance (ANCOVAs) were conducted with a twogroup (intervention or control) comparison and using baseline scores as a covariate to increase statistical power (Rausch, Maxwell, \& Kelley, 2003). For statistically significant tests, effect sizes are reported as partial eta squared values (partial $\eta^{2}$ ) and Cohen's $d$ values. Partial eta-squared values are similar to $\mathrm{R}^{2}$ values and represent the percentage of variance in the outcomes accounted for by the independent variable, for which Cohen (1969) defines small, medium, and large effects as .02, .13, and .16, respectively. Cohen's $d$ values represent the size of the effect as a portion of the pooled 
standard deviation, for which Cohen (1969) defines small, medium, and large effects as $0.20,0.50$, and 0.80 , respectively.

\section{Results}

Sample characteristics. Demographic characteristics for the intent-to-treat, treatment completer, and control groups are provided in Table 6. The intent-to-treat (ITT) group is made up of all participants assigned to the LEAP intervention, irrespective of whether they participated in any portion of the intervention. The group designated treatment completers (TxComplete) refers to a subset of the ITT group, specifically those that accessed both of the LEAP modules and reported completing at least one of the activities scheduled as part of the Assess Your Options and Plan phases of the intervention. There were no significant differences between study groups on any demographic variables. Means and standard deviations of study measures for the overall sample, as well as for ITT, TxComplete, and control groups, are provided in Table 7. At baseline, independent samples $t$-tests revealed no significant differences between the ITT and control group for any baseline clinical variables. Similarly, there were no significant differences between the ITT and TxComplete groups for any baseline clinical variables. However, treatment completers reported a significantly lower average suicidal ideation score at baseline, when compared to the control group, $t(50.59)=-2.54, p=.01$. 
Table 6. Demographic Characteristics of ITT, TxComplete, and Control Groups

\begin{tabular}{|c|c|c|c|}
\hline & ITT & TxComplete & Control \\
\hline & Group & Group & Group \\
\hline & $(n=41)$ & $(n=18)$ & $(n=39)$ \\
\hline Age & $16.95(1.61)$ & $17.39(1.54)$ & $16.90(1.73)$ \\
\hline Female & $70.7 \%$ & $72.2 \%$ & $66.7 \%$ \\
\hline Hispanic & $73.2 \%$ & $77.8 \%$ & $56.4 \%$ \\
\hline \multicolumn{4}{|l|}{ Race } \\
\hline White & $65.9 \%$ & $77.8 \%$ & $69.2 \%$ \\
\hline African American/Black & $12.2 \%$ & $5.6 \%$ & $20.5 \%$ \\
\hline Asian & $9.8 \%$ & $16.7 \%$ & $5.1 \%$ \\
\hline Native Hawaiian/Pacific Islander & $0.0 \%$ & $0.0 \%$ & $0.0 \%$ \\
\hline Native American/AK Native & $2.4 \%$ & $5.6 \%$ & $0.0 \%$ \\
\hline Other & $9.8 \%$ & $0.0 \%$ & $7.7 \%$ \\
\hline Born in the United States & $73.2 \%$ & $66.7 \%$ & $66.7 \%$ \\
\hline Eligible for Free Lunch & $48.8 \%$ & $61.1 \%$ & $64.1 \%$ \\
\hline Heterosexual & $92.7 \%$ & $88.9 \%$ & $84.6 \%$ \\
\hline
\end{tabular}

Aim 1: Participant completion of and satisfaction with LEAP. Of the 41 participants assigned to the LEAP intervention, $92.7 \%(n=38)$ completed one LEAP module and $61.0 \%(n=25)$ completed both LEAP modules. With regard to the activities scheduled while completing the modules, $68.3 \%(n=28)$ reported completing at least one 
activity. The mean number of activities completed was 2.50 (SD 1.89). In total, 18 participants (43.9\%) were designated "treatment completers," having completed both LEAP modules and at least one of the activities they scheduled. In addition, $85.4 \%$ ( $n=$ 35) of those assigned to the LEAP intervention reported that they believed they could use the principles taught by the LEAP program on their own.

With regard to participant satisfaction, mean SSS scores among intent-to-treat, treatment completer, and control groups were not significantly different at either post intervention or follow-up assessments. Independent samples $t$-tests revealed no significant differences between SSS scores across intervention and control group participants, or across treatment completers and control group participants. 
Table 7. Means and Standard Deviations of Pilot Randomized Controlled Trial Study Measures

\begin{tabular}{|c|c|c|c|c|}
\hline & Full Sample & Control Group & ITT Group & TxComplete \\
\hline & Mean (SD) & Mean (SD) & Mean (SD) & Mean (SD) \\
\hline & $(n=80)$ & $(n=39)$ & $(n=41)$ & $(n=18)$ \\
\hline \multicolumn{5}{|l|}{ Baseline } \\
\hline Perceived burdensomeness & $19.90(5.71)$ & $19.67(5.36)$ & $20.12(6.08)$ & $21.17(6.11)$ \\
\hline Suicidal ideation & $5.14(5.39)$ & $6.15(6.00)^{\mathrm{b}}$ & $4.17(4.60)$ & $2.78(3.66)^{\mathrm{b}}$ \\
\hline Thwarted belongingness & $37.08(7.38)$ & $37.97(7.70)$ & $36.22(7.04)$ & $38.50(6.29)$ \\
\hline Depressive symptoms & $26.33(4.17)$ & $26.85(4.32)$ & $25.83(4.01)$ & $26.22(3.70)$ \\
\hline \multicolumn{5}{|l|}{ Post-intervention } \\
\hline Perceived burdensomeness & $18.27(6.30)$ & $18.81(6.26)^{\mathrm{b}}$ & $17.76(6.37)$ & $15.94(5.83)^{b}$ \\
\hline Suicidal ideation & $3.24(4.93)$ & $4.49(6.01)$ & $2.05(3.27)$ & $1.50(2.66)$ \\
\hline Thwarted belongingness & $33.46(8.10)$ & $35.22(8.60)$ & $31.78(7.32)$ & $31.78(7.17)$ \\
\hline Depressive symptoms & $23.86(5.25)$ & $24.64(5.90)$ & $23.12(4.50)$ & $22.11(4.03)$ \\
\hline Satisfaction with Services & $8.76(1.84)$ & $8.89(1.86)$ & $8.64(1.84)$ & $8.72(1.64)$ \\
\hline \multicolumn{5}{|l|}{ Follow-up } \\
\hline Perceived burdensomeness & $14.85(6.60)$ & $15.85(6.25)^{\mathrm{b}}$ & $13.90(6.86)$ & $10.93(6.46)^{\mathrm{b}}$ \\
\hline Suicidal ideation & $2.21(3.75)$ & $2.57(4.40)$ & $1.69(3.01)$ & $1.37(2.93)$ \\
\hline Thwarted belongingness & $29.48(8.51)$ & $31.76(8.09)^{\mathrm{ab}}$ & $27.30(8.42)^{\mathrm{a}}$ & $24.67(9.16)^{b}$ \\
\hline Depressive symptoms & $22.08(5.07)$ & $23.00(5.41)^{\mathrm{b}}$ & $20.93(4.49)$ & $19.65(4.24)^{b}$ \\
\hline Satisfaction with Services & $9.19(2.14)$ & $9.27(1.64)$ & $9.11(2.55)$ & $9.43(2.39)$ \\
\hline
\end{tabular}

Aim 2: Efficacy of the LEAP intervention. In order to examine the efficacy of the LEAP intervention, a series of ANCOVA analyses were conducted with baseline 
scores entered as covariates. The initial efficacy tests were conducted comparing treatment completers to the control group. Additional analyses were then conducted as intent-to-treat analyses, utilizing the full intervention and control groups.

When comparing treatment completers $(n=18)$ to those in the control group, treatment completers did report significantly lower perceived burdensomeness scores at post-intervention as compared to those in the control condition, controlling for baseline perceived burdensomeness scores, $\mathrm{F}(2,55)=6.18 p=.02$, partial $\eta^{2}=0.10, d=0.47$. However, treatment completers did not report significantly lower suicidal ideation scores, $\mathrm{F}(2,55)=0.62, p=.44$, depressive symptom scores, $\mathrm{F}(2,55)=2.77, p=.10$, or thwarted belongingness scores, $\mathrm{F}(2,55)=3.55, p=.07$, at post-intervention as compared to those in the control condition, controlling for the respective baseline scores.

For the primary outcome of perceived burdensomeness, participants in the LEAP intervention condition did not report significantly lower perceived burdensomeness scores at post-intervention as compared to those in the control condition, controlling for baseline perceived burdensomeness scores, $\mathrm{F}(2,78)=1.31, p=.26$. Similarly, for the secondary outcome of suicidal ideation, participants in the LEAP intervention condition did not report significantly lower suicidal ideation scores at post-intervention as compared to those in the control condition, controlling for baseline suicidal ideation scores, $\mathrm{F}(2,78)=2.42, p=.12$. For the tertiary outcomes of depressive symptoms and thwarted belongingness, participants in the LEAP intervention condition did not report significantly lower depressive symptom scores, $\mathrm{F}(2,78)=0.58, p=.45$, or thwarted belongingness scores, $\mathrm{F}(2,78)=2.53, p=.12$, at post-intervention as compared to those in the control condition, controlling for the respective baseline scores. 
Aim 3: Maintenance of LEAP intervention effects. In order to examine the maintenance of the LEAP intervention effects, a series of ANCOVAs were conducted with baseline scores entered as covariates. The initial tests were conducted comparing treatment completers to the control group. Additional analyses were then conducted as intent-to-treat analyses, utilizing the full intervention and control groups.

When comparing treatment completers $(n=18)$ to those in the control group, treatment completers reported significantly lower perceived burdensomeness scores at follow-up as compared to those in the control condition, controlling for baseline perceived burdensomeness scores, $\mathrm{F}(2,55)=14.59, p<.001$, partial $\eta^{2}=0.21, d=0.77$. However, treatment completers did not report significantly lower suicidal ideation scores at follow-up as compared to those in the control condition, controlling for baseline suicidal ideation scores, $\mathrm{F}(2,55)=0.25, p=.62$. For the tertiary outcomes of depressive symptoms and thwarted belongingness, treatment completers condition reported significantly lower depressive symptom scores $\left(\mathrm{F}(2,55)=7.18, p=.01\right.$, partial $\eta^{2}=0.12$, $d=0.69)$ and thwarted belongingness scores $\left(\mathrm{F}(2,55)=10.08, p=.002\right.$, partial $\eta^{2}=$ $0.16, d=0.82)$ at follow-up as compared to those in the control condition, controlling for the respective baseline scores.

For the primary outcome of perceived burdensomeness, in intent-to-treat analyses, participants in the LEAP intervention condition did not report significantly lower perceived burdensomeness scores at follow-up as compared to those in the control condition, controlling for baseline perceived burdensomeness scores, $\mathrm{F}(2,78)=2.83, p=$ .10. Similarly, participants in the LEAP intervention condition did not report significantly lower suicidal ideation scores, $\mathrm{F}(2,78)=0.1 p=.92$, or depressive symptom 
scores, $\mathrm{F}(2,78)=3.32, p=.07$, at follow-up as compared to those in the control condition. Participants in the intent-to-treat group did report lower thwarted belongingness scores, $\mathrm{F}(2,78)=4.64, p=.03$, partial $\eta^{2}=0.06, d=0.54$, at follow-up as compared to those in the control condition, controlling for baseline thwarted belongingness scores.

\section{Discussion}

The present study was a pilot randomized controlled trial of a new, web-based brief intervention called LEAP, designed to reduce perceptions of burdensomeness among adolescents who reported an elevated level of burdensome cognitions. This pilot RCT was intended to evaluate participant satisfaction with LEAP and participant use of LEAP modules. It also examined whether the LEAP program produced significantly lower levels of perceived burdensomeness, suicidal ideation, depressive symptoms, and thwarted belongingness at post-intervention and six-week follow-up assessments.

Aim 1: Participant completion of satisfaction with LEAP. The purpose of Aim 1 was to provide additional evidence of the acceptability of the LEAP program to adolescents. While the majority of participants randomized to the LEAP intervention condition completed the first intervention module, approximately $2 / 3$ completed both LEAP modules and fewer than half were designated treatment completers, having completed both LEAP modules and at least one of the activities planned during the intervention. Thus, while a sizeable portion engaged the intervention, more than half of those randomized to the intervention did not complete it, indicating room for improvement in engaging adolescents in the LEAP intervention process. Of note, 
adolescents in this study were recruited from the community and were not a treatmentseeking sample, making it difficult to compare this rate of intervention completion to traditional intervention studies. The addition of reminders to complete the second LEAP module resulted in a completion rate of $61 \%$, a considerable improvement over the open trial, in which only $25 \%$ completed the second intervention module.

While adolescents were reminded to complete the second intervention module, no prompts or reminders were available for completing the scheduled activities, which may partially explain the low rate of completion for these activities. Additional efforts to refine the LEAP intervention should take into account the low rate of intervention engagement and make efforts to enhance adolescent engagement in the intervention modules and follow-through with activities planned during the intervention.

Overall, participants in both intervention and control conditions reported satisfaction with the LEAP intervention or resource list, respectively. Similar satisfaction scores across groups indicate that the LEAP intervention is not viewed by participants more favorably than the resource list. Nor was the task of completing the computer modules viewed unfavorably. Given that the LEAP intervention did not receive a higher average satisfaction rating than the control condition, future work may consider modifications to the LEAP program to increase participant satisfaction.

These findings together indicate the need for additional efforts to engage adolescents in the LEAP intervention. It is unclear, based on the results of this study, the reasons for participants' failure to complete both intervention modules and/or to complete their planned activities. It is possible that efforts to enhance participant "buy in" to the intervention may result in a higher rate of intervention completion. Another possibility is 
that changes to the intervention medium (e.g., a mobile phone application as opposed to a web-based survey) might allow for more seamless incorporation of automated scheduling for activity completion and text-message reminders of upcoming scheduled activities and modules. If failure to complete scheduled activities occurred, at least in part, from participants forgetting to complete them, a mobile application or similar format with automated prompts may substantially increase follow-through and intervention completion. However, given that the LEAP intervention could be made available to a large number of adolescents at very little cost, the low rate of completion may not prove a critical flaw to producing clinically significant intervention effects.

Aim 2: Efficacy of the LEAP intervention. Overall intent-to-treat analyses of the intervention effects showed no significant impact of the LEAP intervention as compared to the control condition. Analyses of treatment completers, a subset of those randomized to the LEAP intervention condition, who completed both intervention modules and at least one scheduled activity, demonstrated significant reductions in perceived burdensomeness scores, as compared to the control condition. These results are promising, as they indicate a significant reduction in perceived burdensomeness for those who engaged in the LEAP intervention. That is, while treatment completers represented just under half of those randomly assigned to the LEAP intervention, those who did complete the LEAP intervention reported reduced perceived burdensomeness at both post-intervention and follow-up assessments, compared to adolescents in the control group. Thus, adolescents who completed the LEAP intervention appear to have benefitted relative to those who did not receive the LEAP intervention. 
No intervention effects were found at post-intervention for the secondary outcome, suicidal ideation. This may be due, in part, to the decrease in suicidal ideation scores across groups. It may also reflect the low rate of suicidal ideation in the sample, as suicidal ideation was not an inclusion criterion for the present study and a high level of suicidal ideation was an exclusion criterion. In addition, the treatment completer group had a low mean suicidal ideation score at baseline, making statistically significant improvement unlikely. The LEAP intervention, however, was designed not only to reduce current suicidal ideation but to prevent or attenuate the occurrence of suicidal ideation in the future. As a result, a significant short-term intervention effect on suicidal ideation may not be critical for the success of the LEAP intervention. Rather, a potential intervention effect in which LEAP engagement prevents the development of suicidal ideation would demonstrate the efficacy of the LEAP intervention. Such an impact would require a much larger sample size and greater study duration to detect statistically significant effects than was possible in this pilot trial.

In addition, no intervention effects were reported at post-intervention for depressive symptoms or thwarted belongingness. This is not particularly surprising, as the LEAP intervention is not designed to target either of these constructs directly and so short term pre-to-post intervention effects would be unexpected.

Aim 3: Maintenance of LEAP intervention effects. Overall intent-to-treat analyses of intervention effects at six-week follow-up showed no significant impact of the LEAP intervention on perceived burdensomeness, suicidal ideation, or depressive symptoms, but did reveal a significant reduction in thwarted belongingness among those randomized to the LEAP intervention, as compared to those in the control condition. In 
addition, comparison of treatment completers and controls yielded significant positive effects on perceived burdensomeness, thwarted belongingness, and depressive symptoms at six-week follow-up. It appears that the LEAP intervention had a delayed impact on perceived burdensomeness (as the effect size increased from post intervention to followup), as well as on thwarted belongingness and depressive symptoms. Though no intervention effect on suicidal ideation was found, it is important to note that both the intervention and control groups reported very little suicidal ideation at the follow-up assessment, which may have limited the ability to detect significant differences between the two groups.

While the LEAP intervention program specifically targeted burdensome cognitions, it did so in the context of a participant-selected target relationship. That is, the Explore, Assess Your Options, and Plan phases, including all activity scheduling within the intervention, are focused on an adolescent-identified target individual. While it is not possible to identify the means by which the LEAP intervention led to significant reductions in thwarted belongingness and depressive symptoms, one possibility is that the initial focus on a single target individual, and the reduction of perceptions of burdensomeness on that target individual, led to a wider shift in the target-adolescent relationship.

For example, if an adolescent selected his mother as the target of the intervention, he would have planned activities to contribute to his mother's life and a discussion with his mother regarding his burdensome cognitions. If the adolescent subsequently discussed his burdensome cognitions with his mother and engaged in activities to contribute to her well-being, this may have led to fewer negative and/or more frequent 
positive mother-adolescent experiences, resulting in better overall relationship quality. This may also have increased his mother's attention to the issue and prompted her to respond with positive verbalizations and behaviors. These positive experiences may have, in turn, yielded reduced perceptions of thwarted belongingness and an increase in positive, rewarding experiences, resulting in fewer depressive symptoms. While it is not possible to examine these hypothetical mechanisms of change in the current study, it will be important for future research to consider possible mechanisms by which the LEAP intervention may have impacted depressive symptoms and thwarted belongingness.

Strengths and limitations of the present study. Strengths of the study include the use of a an ethnic minority sample, the randomized design, the brief nature of the intervention, and the high rate of completion of post-intervention and follow-up assessments. Future research should build on these strengths while addressing the weaknesses noted below.

It is important to consider the results of this study in light of the study limitations. The results of this study should be generalized with caution, as the sample was primarily female and Hispanic. Further, the recruitment methods utilized in this study did not ensure that the sample was representative of the larger population of adolescents in the study recruitment area. Further research is needed to address the issue of generalizability to other adolescent populations.

Also of note, the treatment completer group reported significantly lower suicidal ideation scores at baseline than the control group. It is not possible to determine from the available data why this occurred: One possibility is that individuals experiencing greater suicidal ideation were too greatly impaired to be willing or able to complete the 
intervention modules and/or the activities planned within the modules. Limited participant engagement in the intervention makes it difficult to determine if the intervention would be effective even for those with elevated suicidal ideation, if they were to complete it. Additional efforts to enhance participant engagement with the intervention may be needed to address this issue. Alternatively, failure of those with elevated suicidal ideation to complete the intervention may imply that the LEAP intervention needs to be more selectively implemented as a prevention program, prior to the onset of suicidal ideation. Future research will need to be cognizant of this issue and examine predictors of participant completion more closely.

Another important limitation was the lack of participant blindness to the intervention condition. It is possible that some participants benefitted simply from knowing that they were receiving an intervention. A more stringent examination of the efficacy of the LEAP intervention should include a comparison intervention similar in administration format and time required. Utilizing a comparison condition provided in the same technological format and requiring a similar amount of time, but without active cognitive-behavioral intervention components, would allow for more rigorously concluding that aspects of the LEAP intervention, specifically, result in changes in perceived burdensomeness.

Conclusions. The present study was a pilot RCT of LEAP, a brief, web-based prevention program to reduce perceptions of burdensomeness among adolescents. Results indicated that adolescents found the LEAP program acceptable. While only $45 \%$ of adolescents randomized to the LEAP intervention successfully completed treatment, those who did so reported significantly lower perceived burdensomeness scores at post- 
intervention than those randomized to the control condition. Further, those who completed treatment reported significantly lower perceived burdensomeness, thwarted belongingness, and depressive symptom scores at a six-week follow-up assessment than did those in the control condition. Results indicate room for improvement in adolescents' completion of the intervention. Overall, however, those who completed the LEAP intervention showed significant reductions in perceived burdensomeness. The LEAP intervention shows promise as a low-cost, prevention program for reducing perceived burdensomeness among adolescents. 


\section{GENERAL DISCUSSION}

\section{Summary of the Present Work}

Suicide is the third leading cause of death among 15-24 year olds in the United States (Centers for Disease Control and Prevention, 2014) and suicide attempts and suicidal ideation occur frequently in adolescence (Centers for Disease Control and Prevention, 2012). Thus, further development of prevention programs to reduce suiciderelated behaviors is needed, especially those focusing on non-clinical suicide risk management (Suicide Prevention Resource Center and SPAN USA, 2010).

To date, however, most adolescent suicide prevention programs focus either on universal approaches such as case identification or increasing public knowledge about warning signs of suicide (e.g., Wyman et al., 2008) or indicated approaches to reduce risk in adolescents reporting serious suicidal ideation or suicide attempts (e.g., King et al., 2009). These approaches have rarely demonstrated sustained reductions in adolescent suicide-related behaviors (e.g., King, et al., 2009; King, et al., 2006; Wyman et al., 2010) though some programs have shown promising results (e.g., Esposito-Smythers et al., 2011; Fleishaker, 2011). Existing programs with empirical support are highly intensive in nature and are geared toward acutely suicidal adolescents. A review of the extant literature on prevention science for adolescent suicide-related behaviors identified a need for brief, low cost approaches to address non-clinical suicide risk. This led to the development and initial examination of the LEAP intervention.

The LEAP program is a brief, web-based, cognitive-behavioral prevention program designed to reduce cognitions of burdensomeness among adolescents. The LEAP intervention is designed to overcome barriers to dissemination by requiring no 
direct contact from a mental health professional and by utilizing a web-based format with the potential for broad dissemination. In the LEAP intervention, adolescents with elevated levels of perceived burdensomeness are directed to two online modules that provide a guided experience of psychoeducation, exploration of burdensome cognitions, and the use of cognitive and behavioral strategies to reduce perceived burdensomeness.

First, an open trial of the LEAP program was conducted to examine the feasibility of the study protocol, generate feedback regarding the LEAP program modules, and refine the program modules. Results of the open trial indicated high feasibility of the intervention protocol but also identified opportunities for improvement in adolescent completion of the intervention. Based on the results of the open trial and feedback from participants, modifications were made to the LEAP modules and study protocol.

Subsequently, a pilot RCT of the program was conducted to examine participant satisfaction with the intervention and to provide initial evidence for its efficacy. While fewer than half of those randomized to the LEAP intervention were designated treatment completers, initial evidence points toward the efficacy of the LEAP intervention for reducing perceived burdensomeness among treatment completers and for maintenance of the intervention effect six weeks later. Additionally, the LEAP intervention resulted in significant reductions in depressive symptoms and thwarted belongingness at a six-week follow-up.

\section{Future Directions}

These two studies provide initial evidence for the feasibility and acceptability of the LEAP intervention, as well as initial evidence of its efficacy at post-intervention and maintenance of the intervention effect at six-week follow-up. As the LEAP intervention 
was specifically designed not to require the involvement of a mental health professional, the intervention is well-positioned for implementation and evaluation within a variety of contexts. Future research on the efficacy of LEAP should make use of this unique aspect of the program by integrating the LEAP intervention into a more naturalistic, communitybased setting, allowing simultaneous examination of the efficacy and effectiveness of the intervention. Potential settings in which the program may be integrated include schoolbased settings, primary care centers, and emergency departments, among others - all places where adolescents frequent (schools) or have long wait times (primary care centers and emergency departments), allowing sufficient time for screening and module completion. In schools, for example, students could be prompted to complete a voluntary perceived burdensomeness screen at the start of each quarter - allowing for multiple opportunities to identify at risk students and provide the LEAP intervention.

The LEAP intervention could also be implemented as an adjunctive or adjuvant intervention in existing mental health care facilities. For example, as an adjunctive intervention, adolescents awaiting the start of treatment who report elevated perceived burdensomeness in a brief screen could begin the online modules prior to starting other treatment protocols. Alternatively, as an adjuvant intervention, adolescents completing psychosocial treatment for mental health issues known to be associated with suiciderelated behaviors could be provided the LEAP modules as an adjuvant intervention experience and suicide prevention initiative.

Future work should also consider possible avenues for increasing completion of the LEAP intervention modules and follow-through with planned activities. Given that only approximately half of participants assigned to the LEAP intervention completed the 
intervention in the pilot randomized controlled trial, there is a need for improvement in this aspect of the intervention. One possibility for enhancing intervention completion is the addition of automated reminders for intervention modules and scheduled activities. This could be achieved by utilizing smart phone technology, such as a smart phone application that allows planned events to be integrated with mobile calendars or GPS triggers. In this way, adolescents could be reminded to engage in their planned activities at specified times or in predetermined locations. For example, an adolescent who identifies a behavior to be completed "when I get home from school," could receive a calendar-based reminder activated at 4:00pm or a GPS-based reminder activated when the adolescent's phone recognizes that he has arrived at home. Both of these options are within the abilities of current smartphone technology.

Utilization of smart phone technology may also allow researchers to collect richer data to track the type, time, and rate of activity and module completion. Future research should consider the type and number of activities completed to better examine the extent of intervention engagement necessary to produce reductions in perceived burdensomeness and the relative utility of the two cognitive-behavioral intervention skills utilized in the LEAP intervention. Accurate records of the type of behaviors adolescents engage in after completing the intervention modules (challenging negative cognitions versus engaging in contributing activities) may provide support for greater efficacy associated with one cognitive-behavioral skill over the other, allowing for refinement of the intervention modules. This methodology would also allow for careful examination of potential mediators of the effect of intervention on perceived burdensomeness (and/or suicidal ideation). The frequency and type of activities completed following the 
introduction of the LEAP modules may mediate the effect of the intervention and shed light on means by which the LEAP intervention influences perceived burdensomeness.

Future research should also consider methods for measuring the impact of repeated use of the LEAP intervention to determine if a dose-response relationship is present. That is, adolescents could be repeatedly screened for elevated perceived burdensomeness at regular intervals and prompted to participate in the LEAP intervention at each point where elevated levels of perceived burdensomeness are present. In this way it would be possible to identify if repeated engagement in the LEAP intervention results in more pronounced intervention effects or enhanced maintenance of intervention effects over extended follow-up periods.

Finally, it will be important for future research to consider the potential impact of the LEAP intervention on the presence of suicide-related behaviors. Identification of a reduced rate of suicidal ideation as a result of the LEAP intervention may require large sample sizes and long-term or intensive follow-up protocols, with sufficient power to detect small differences in the frequency or intensity of suicidal ideation across intervention and control groups. As with other prevention efforts, one challenge to broad dissemination will be demonstrating efficacy on suicide-related outcomes, in addition to impacts on theoretically-related risk factors. Broad adoption of the LEAP intervention will require careful attention to this issue and the documentation of significant impacts on suicide-related outcomes.

\section{Conclusions}

A review of the extant literature on the state of prevention science for adolescent suicide-related behaviors led to the development and initial examination of the LEAP 
intervention. Study 1 was an open trial of the LEAP intervention. Results indicated strong feasibility of the study protocol for recruiting and retaining adolescent participants. A number of modifications were made to the intervention modules based on feedback provided by the participants and examination of participants' responses within the intervention modules.

Study 2 was a pilot randomized controlled trial of LEAP. Results indicated that those who completed the LEAP intervention reported significantly lower perceived burdensomeness scores at post-intervention than those in the control condition. Further, those who completed the intervention reported significantly lower perceived burdensomeness, thwarted belongingness, and depressive symptom scores at follow-up than did those in the control condition. No significant effects on suicidal ideation were noted. Overall, the LEAP intervention shows promise as a low-cost, prevention program for reducing perceived burdensomeness among adolescents. 


\section{LIST OF REFERENCES}

Ajzen, I. (1991). The Theory of Planned Behavior. Organizational Behavior and Human Decision Processes, 50, 179-211. doi: 10.1016/0749-5978(91)90020-T

American Psychological Association. (2015). Society of Clinical Psychology, American Psychological Assocaition, Division 12. Retrieved from www.div12.org/PsychologicalTreatments/treatment/bpd_dbt.html.

Anestis, M. D., Bagge, C. L., Tull, M. T., \& Joiner, T. E. (2011). Clarifying the role of emotion dysregulation in the interpersonal-psychological theory of suicidal behavior in an undergraduate sample. Journal of Psychiatric Research, 45(5), 603-611. doi: 10.1037/0033-295x.96.2.358

Anestis, M. D., \& Joiner, T. E. (2011). Examining the role of emotion in suicidality: Negative urgency as an amplifier of the relationship between components of the interpersonal-psychological theory of suicidal behavior and lifetime number of suicide attempts. Journal of Affective Disorders, 129(1-3), 261-269. doi: 10.1016/j.brat.2007.08.012

Arias, S. A., Zhang, Z., Hillerns, C., Sullivan, A. F., Boudreaux, E. D., Miller, I., \& Camargo, C. A. (2014). Using structured telephone follow-up assessments to improve suicide-related adverse event detection. Suicide and Life-Threatening Behavior, 44(5), 537-547. doi: 10.1111/sltb.12088

Asarnow, J. R. \& Miranda, J. (2014). Improving care for depression and suicide risk in adolescents: Innovative strategies for bringing treatments to community settings. Annual Review of Clinical Psychology, 10, 275-303. doi: 10.1146/annrev-clinpsy032813-153742

Athay, M. M., \& Bickman, L. (2012). Development and psychometric evaluation of the youth and caregiver service satisfaction scale. Administration and Policy in Mental Health and Mental Health Services Research, 39(1-2), 71-77. doi: 10.1007/s10488-012-0407-y

Barzilay, S. \& Apter, A. (2014). Psychological models of suicide. Archives of Suicide Research, 18, 295-312. doi: 10.1080/13811118.2013.824825.

Baumeister, R. F. (1990). Suicide as escape from self. Psychological Review, 97(1), 9090-113. doi: 10.1037/0033-295x.97.1.90

Baumeister, R. F., \& Leary, M. R. (1995). The need to belong: Desire for interpersonal attachments as a fundamental human motivation. Psychological Bulletin, 117(3), 497-529. doi: 10.1037/0033-2909.117.3.497 
Beck, A. T., Kovacs, M., \& Weissman, A. (1979). Assessment of suicidal ideation: The Scale for Suicide Ideation. Journal of Consulting and Clinical Psychology, 47(2), $343-352$.

Beck, A. T., Rush, A. J., Shaw, B. F., \& Emery, G. (1979). Cognitive therapy of depression. Guildford Press: New York.

Beck, A. T., Steer, R. A., Beck, J. S., \& Newman, C. F. (1993). Hopelessness, depression, suicidal ideation, and clinical diagnosis of depression. Suicide and Life-Threatening Behavior, 23(2), 139-145.

Beck, A. T., Steer, R. A., Kovacs, M., \& Garrison, B. (1985). Hopelessness and eventual suicide: A 10-year prospective study of patients hospitalized with suicidal ideation. The American Journal of Psychiatry, 142(5), 559-563.

Beck, J. S., Liese, B. S., \& Najavits, L. M. (2005). Cognitive therapy. In Eds. R. J. Frances, S. I. Miller, \& A. H. Mack. Clinical textbook of addictive disorders. Guilford Press: New York.

Bennett, G. G. \& Glasgow, R. E. (2009). The delivery of public health interventions via the internet: Actualizing their potential. Annual Review of Public Health, 30, 273292. doi: 10.1146/annurev.publhealth.031308.100235.

Bickman, L., Athay, M. M., Riemer, M., Lambert, E. W., Kelley, S. D., Breda, C., ... Vides de Andrade, A. R. (2010). Manual of the Peabody Treatment Progress Battery, 2nd ed. Retrieved from http://peabody.vanderbilt.edu/ptpbl

Brent, D. A., Perper, J. A. J., Moritz, G. G., Allman, C. C., Friend, A. A., Roth, C. C., , . Baugher, M. M. (1993). Psychiatric risk factors for adolescent suicide: A casecontrol study. Journal of the American Academy of Child \& Adolescent Psychiatry, 32(3), 521-529. doi: 10.1097/00004583-1993305000-00006

Bryan, C. J. (2011). The clinical utility of a brief measure of perceived burdensomeness and thwarted belongingness for the detection of suicidal military personnel. Journal of Clinical Psychology, 67(10), 981-992. doi: 10.1016/00057967(96)00033-2

Bryan, C. J., Clemans, T. A., \& Hernandez, A. M. (2012). Perceived burdensomeness, fearlessness of death, and suicidality among deployed military personnel.

Personality and Individual Differences, 52, 374-379. doi: 10.1016/j.paid.2011.10.045 
Bryan, C. J., Cukrowicz, K. C., West, C. L., \& Morrow, C. E. (2010). Combat experience and the acquired capability for suicide. Journal of Clinical Psychology, 66(10), 1044-1056. doi: 10.1002/jclp.20703

Bryan, C. J., Morrow, C., Anestis, M., \& Joiner, T. (2010). A preliminary test of the interpersonal-psychological theory of suicidal behavior in a military sample.

Personality and Individual Differences, 48(3), 347-350. doi:

10.1016/j.paid.2009.10.023

Bryan, C. J., Ray-Sannerud, B., Morrow, C. E., \& Etienne, N. (2012). Guilt is more strongly associated with suicidal ideation among military personnel with direct combat exposure. Journal of Affective Disorders. doi: 10.1016/j.jad/2012.11.044

Calear, A. L., Christensen, H., Mackinnon, A., Griffiths, K. M., \& O'Kearney, R. (2009). The YouthMood Project: A cluster randomized controlled trial of an online cognitive behavioral program with adolescents. Journal of Consulting and Clinical Psychology, 77(6), 1021-1032. doi: 10.1037/a0017391

Carter, G., Clover, K., Whyte, I., Dawson, A., \& D'Este, C. (2007). Postcards from the Edge: 24-month outcomes of a randomised controlled trial for hospital-treated self-poisoning. British Journal of Psychiatry, 191, 548-553. doi: 10.1192/bjp.bp.107.038406

Centers for Disease Control and Prevention. (2014a). Injury prevention \& control: Data \& statistics (WISQARS). Retrieved March 1, 2014, from http://www.cdc.gov/injury/wisqars/index.html.

Centers for Disease Control and Prevention. (2014b). Youth risk behavior surveillance United States, 2011. MMWR, 61(4)

Chen, W., Shyu, S., Lin, G., Chen, C., Ho, C., Lee, M., \& Chou, F. H. (2013). The predictors of suicidality in previous suicide attempters following case management services. Suicide and Life-Threatening Behavior, 43(5), 469-478. doi: $10.1111 /$ sltb.12031

Chen, Y., Wu, K. C., Yousuf, S., \& Yip, P. S. F. (2012). Suicide in Asia: Opportunities and challenges. Epidemiology Review, 34(1), 129-144. doi: 10.1093/epirev/mxr025

Clarke, G., Lewinsohn, P., \& Hops, H. (1990). Leaders manual for adolescent groups: Adolescent Coping with Depression course. Kaiser Permanente: Portland, OR.

Cohen, J. (1969). Statistical power analysis for the behavioral sciences. $2^{\text {nd }}$ edition. Lawrence Erlbaum Associates, Hillsdale, NJ. 
Cohen, J., Cohen, P., West, S. G., \& Aiken, L. S. (2003). Applied multiple regression/correlation analysis for the behavioral sciences. $3^{\text {rd }}$ edition. Lawrence Erlbaum Associates, Inc., Mahwah, New Jersey.

Cox, D. W., Ghahramanlou-Holloway, M., Greene, F. N., Bakalar, J. L., Schendel, C. L., Nademin, M. E., . . Kindt, M. (2011). Suicide in the United States Air Force: Risk factors communicated before and at death. Journal of Affective Disorders, 133, 398-405. doi: 10.1016/j.jad.2011.05.011

Crosby, A. E., Ortega, L., \& Melanson, C. (2011). Self-directed violence: Uniform definitions and recommended data elements, version 1.0. Centers for Disease Control and Prevention, National Center for Injury Prevention and Control: Atlanta, GA.

Cuijpers, P., van Straten, A., \& Warmerdam, L. (2007). Behavioral activation treatments of depression: A meta-analysis. Clinical Psychology Review, 27, 318-326. doi: 10.1016/j.cpr.2006.11.001.

Davidson, C. L., Wingate, L. R., Rasmussen, K. A., \& Slish, M. L. (2009). Hope as a predictor of interpersonal suicide risk. Suicide and Life-Threatening Behavior, 39(5), 499-507. doi: 10.1146/annurev.psych.56.091103.070320

Davidson, C. L., Wingate, L. R., Grant, D. M., Judah, M. R., \& Mills, A. C. (2011). Interpersonal suicide risk and ideation: The influence of depression and social anxiety. Journal of Social and Clinical Psychology, 30(8), 842-855. doi: $10.1521 /$ jscp.2011.30.8.842

Deci, E. L., \& Ryan, R. M. (2000). The "what" and "why" of goal pursuits: Human needs and the self-determination of behavior. Psychological Inquiry, 11(4), 227-268. doi: 10.1207/s15327965pli1104_01

Deci, E. L., \& Ryan, R. M. (2008). Self-determination theory: A macrotheory of human motivation, development, and health. Canadian Psychology/Psychologie canadienne, 49(3), 182-185. doi: 10.1037/0022-3514.84.4.822

Donaldson, D., Spirito, A., \& Esposito-Smythers, C. (2005). Treatment for adolescents following a suicide attempt: Results of a pilot trial. Journal of the American Academy of Child and Adolescent Psychiatry, 44(2), 113-120. doi: 10.1097/00004583-200502000-00003

Durkheim, E. (1897). Le suicide: Etude de sociologie. Paris: Alcan.

Eggert, L. L., Thompson, E. A., Herting, J. R., \& Nicholas, L. J. (1995). Reducing suicide potential among high-risk youth: Tests of a school-based prevention 
program. Suicide and Life-Threatening Behavior, 25(2), 276-276-296. doi: 10.1111/j.1943-278X.1995.tb00926.x

Eisenberg, M. E., \& Resnick, M. D. (2006). Suicidality among gay, lesbian and bisexual youth: The role of protective factors. Journal of Adolescent Health, 39(5), 662668. doi: 10.1016/j.jadohealth.2006.04.024

Esposito-Smythers, C., Spirito, A., Kahler, C. W., Hunt, J. \& Monti, P. (2011). Treatment of co-occuring substance abuse and suicidality among adolescents: A randomized trial. Journal of Consulting and Clinical Psychology, 79(6), 728-739. doi: $10.1037 / \mathrm{a} 0026074$

Fleischhaker, C., Bohme, R., Sixt, B., Bruck, C., Schneider, C., \& Schulz, E. (2011). Dialectical Behavioral Therapy for Adolescents (DBT-A): A clinical trial for patients with suicidal and self-injurious behavior and borderline sympotmos with a one-year follow-up. Child and Adolescent Psychiatry and Mental Health, 5(3), 1-10. doi: 10.1186/1753-2000-5-3

Freedenthal, S., Lamis, D. A., Osman, A., Kahlo, D., \& Gutierrez, P. M. (2011). Evaluation of the psychometric properties of the Interpersonal Needs Questionnaire-12 in samples of men and women. Journal of Clinical Psychology, 67(6), 609-623. doi: $10.1002 / j \mathrm{jlp} .20782$

Garza, M. J., \& Pettit, J. W. (2010). Perceived burdensomeness, familism, and suicidal ideation among Mexican women: Enhancing understanding of risk and protective factors. Suicide and Life-Threatening Behavior, 40(6), 561-573. doi: 10.1521/suli.2010.40.6.561

Ghoncheh, R., Kerkhof, A. J. F. M., \& Koot, H. M. (2014). Effectiveness of adolescent suicide prevention e-learning modules that aim to improve knowledge and selfconfidence of gatekeepers: Study protocol for a randomzed controlled trial. Trials, 15(52), 1-7. doi: 10.1186/1745-6215-15-52

Gipson, P. Y., King, C. A., Opperman, K. J., \& Ewell-Foster, C. (2014). Links to Enhancing Teens' Connectedness: A community-based mentorship trial for youth at risk for suicide due to bully victimization, perpetration and/or peer disconnection. Paper presented at the American Association for Suicidology, Los Angeles, CA.

Glasgow, R. E., McKay, H. G., Piette, J. D., \& Reynolds, K. D. (2001). The RE-AIM framework for evaluating interventions: What can it tell us about approaches to chronic illness management? Patient Education and Counseling, 44(2), 119-127. doi: 10.1016/S0738-3991(00)00186-5 
Gould, M. S., Cross, W., Pisani, A. R., Munfakh, J. L., \& Kleinman, M. (2013). Impact of Applied Suicide Intervention Skills Training on the National Suicide Prevention Lifeline. Suicide and Life-Threatening Behavior, 43(6), 678-691. doi: 10.1111/sltb.12049

Gould, M. S., Greenberg, T., Velting, D. M., \& Shaffer, D. (2003). Youth suicide risk and preventive interventions: A review of the past 10 years. Journal of the American Academy of Child \& Adolescent Psychiatry, 42(4), 386-405. doi: 10.1097/01.CHI.0000046821.95464.CF

Greenfield, B., Larson, C., Hechtman, L., Rousseau, C., \& Platt, R. (2002). A rapidresponse outpatient model for reducing hospitalization rates among suicidal adolescents. Psychiatric Services, 53(12), 1574-1579.

Gunn, J. F., Lester, D., Haines, J., \& Williams, C. L. (2012). Thwarted belongingness and perceived burdensomeness in suicide notes. Crisis, 33(3), 178-181. doi: $10.1027 / 0227-5910 / \mathrm{a} 000123$

Halpert, S. C. (2002). Suicidal behavior among gay male youth. Journal of Gay \& Lesbian Psychotherapy, 6(3), 53-79. doi: 10.1300/J236v06n03_07

Hill, R. M., \& Pettit, J. W. (2012). Suicidal ideation and sexual orientation in college students: The roles of perceived burdensomeness, thwarted belongingness, and perceived rejection due to sexual orientation. Suicide and Life-Threatening Behavior, 42(5), 567-579. doi: 10.1111/j.1943.278X.2012.00113.x

Hill, R. M., \& Pettit, J. W. (2013). The role of autonomy in suicidal ideation: Integrating the interpersonal-psychological theory of suicide and self-determination theory. Archives of Suicide Research, 17(3), 288-301. doi: 10.1080/13811118.2013.777001

Hill, R. M., \& Pettit, J. W. (in press). Perceived burdensomeness and suicide-related behaviors in clinical samples: Current evidence and future directions. Journal of Clinical Psychology. doi: 10.1002/jclp.22071

Hill, R. M., Castellanos, D. A., \& Pettit, J. W. (2011). Suicidal behavior and anxiety in children and adolescents: A review of the past 20 years. Clinical Psychology Review, 31, 1133-1144. doi: 10.1016/j.cpr.2011.07.008

Hill, R. M., Rey, Y., Marin, C., \& Pettit, J. W. (unpublished manuscript). Psychometric properties of the Interpersonal Needs Questionnaire: Comparison of five versions in a sample of college students. 
Horwitz, A. G., Hill, R. M., \& King, C. A. (2011). Specific coping behaviors in relation to adolescent depression and suicidal ideation. Journal of Adolescence, 34(5), 1077-1085. doi: 10.1016/s0272-7358(98)00010-5

Huey, S. J., Henggeler, S. W., Rowland, M. D., Halliday-Boykins, C. A., Cunningham, P. B., Pickrel, S. G., \& Edwards, J. (2004). Multisystemic therapy effects on attempted suicide by youths presenting psychiatric emergencies. Journal of the American Academy of Child and Adolescent Psychiatry, 43(2), 183-190. doi: 10.1097/01.chi.0000101700.15837.f3

Isaac, M., Elias, B., Katz, L. Y., Belik, S.-L., Deane, F. P., W. Enns, M., \& Sareen, J. (2009). Gatekeeper training as a preventative intervention for suicide: A systematic review. The Canadian Journal of Psychiatry / La Revue canadienne de psychiatrie, 54(4), 260-260-268.

Jacobson, J. M., Osteen, P. J., Sharpe, J. L., \& Pastoor, J. B. (2012). Randomized trial of a suicide gatekeeper training for social work students. Research on Social Work Practice, 22(3), 270-281. doi: 10.1177/10497315114336015

Jahn, D. R., \& Cukrowicz, K. C. (2011). The impact of the nature of relationships on perceived burdensomeness and suicide ideation in a community sample of older adolescents. Suicide and Life-Threatening Behavior, 41(6), 635-649. doi: 10.1111/j.1943-278X.2011.00060.x

Jahn, D. R., Cukrowicz, K. C., Linton, K., \& Prabhu, F. (2011). The mediating effect of perceived burdensomeness on the relation between depressive symptoms and suicide ideation in a community sample of older adults. Aging \& Mental Health, 15(2), 214-220. doi: 10.1080/13607863.2010.501064

Jobes, D. A. (2012). The Collaborative Assessment and Management of Suicidality (CAMS): An evolving evidence-based clinical approach to suicide risk. Suicide and Life-Threatening Behavior, 42(6), 640-653. doi: 10.1111/j.1943278X.2012.00119.x

Joiner, T. E., Jr. (2005). Why people die by suicide. Cambridge, MA, US: Harvard University Press.

Joiner, T. E., Jr., Pettit, J. W., Walker, R. L., Voelz, Z. R., Cruz, J., Rudd, M. D., \& Lester, D. (2002). Perceived burdensomeness and suicidality: Two studies on the suicide notes of those attempting and those completing suicide. Journal of Social and Clinical Psychology, 21(5), 531-545. doi: 10.1521/jscp.21.5.531.22624

Joiner, T. E., Jr., Van Orden, K., Witte, T., Selby, E., Ribeiro, J., Lewis, R., \& Rudd, M. (2009). Main predictions of the interpersonal-psychological theory of suicidal 
behavior: Empirical tests in two samples of young adults. Journal of Abnormal Psychology, 118(3), 634-646. doi: 10.1037/a0016500

Jordan, J. R., \& McMenamy, J. (2004). Interventions for suicide survivors: A review of the literature. Suicide and Life-Threatening Behavior, 34(4), 337-349. doi: 10.1521/suli.34.4.iii.53739

Katz, L., Cox, B. J., Gunasekara, S., \& Miller, A. L. (2004). Feasibility of Dialectical Behavior Therapy for suicidal adolescent inpatients. Journal of the American Academy of Child \& Adolescent Psychiatry, 43(3), 276-282. doi: 10.1097/00004583-200403000-00008

Kazdin, A. E., \& Blase, S. L. (2011). Rebooting psychotherapy reserach and practice to reduce the burden of mental illness. Perspectives on Psychological Science, 6(1), 21-37. doi: $10.1177 / 1745691610393527$

Kessler, R. C., Berglund, P., Demler, O., Jin, R., Merikangas, K. R., \& Walters, E. E. (2005). Lifetime prevalence and age-of-onset distributions of DSM-IV disorders in the National Comorbidity Survey Replication. Archives of General Psychiatry, 62(6), 593-602. doi: 10.1126/science.280.5365.867.

King, C. A., Gipson, P. Y., \& Horwitz, A. G. (2014). Teen Options for Change: An intervention for adolescent emergency patients who screen positive for suicide risk. Paper presented at the American Association of Suicidology, Los Angeles, CA.

King, C. A., Hill, R. M., Wynne, H., \& Cunningham, R. (2012). Adolescent suicide risk screening: The effect of communication about type of follow-up on adolescents' responses. Journal of Clinical Child and Adolescent Psychology, 41(4), 508-515, doi: 10.1080/15374416.2012.680188

King, C. A., Klaus, N., Kramer, A., Venkataraman, S., Quinlan, P., \& Gillespie, B. (2009). The Youth-Nominated Support Team-Version II for suicidal adolescents: A randomized controlled intervention trial. Journal of Consulting and Clinical Psychology, 77(5), 880-893. doi: 10.1037/a0016552

King, C. A., Kramer, A., Preuss, L., Kerr, D. C. R., Weisse, L., \& Venkataraman, S. (2006). Youth-Nominated Support Team for suicidal adolescents (Version 1): A randomized controlled trial. Journal of Consulting and Clinical Psychology, 74(1), 199-206. doi: 10.1037/0022-006X.74.1.199

Kumar, G., \& Steer, R. A. (1995). Psychosocial correlates of suicidal ideation in adolescent psychiatric inpatients. Suicide and Life-Threatening Behavior, 25(3), 339-346. doi: 11.1111/j.1943-278X.1995.tb00956.x 
Lamis, D. A., \& Lester, D. (2012). Risk factors for suicidal ideation among African American and European American college women. Psychology of Women Quarterly, 36(3), 337-349. doi: 10.1177/0361684312439186

Lamis, D. A., \& Malone, P. S. (2011). Alcohol-related problems and risk of suicide among college students: The mediating roles of belongingness and burdensomeness. Suicide and Life-Threatening Behavior, 41(5), 543-553. doi: 10.1111/j.1943-278X.2011.00052.x

Lancaster, P. G., Moore, J. T., Putter, S. E., Chen, P. Y., Cigularov, K. P., Baaker, A., \& Quinnett, P. (2014). Feasibility of a web-based gatekeeper training: Implications for suicide prevention. Suicide and Life-Threatening Behavior, 44(5), 510-523. doi: $10.1111 /$ sltb. 12086

Latimer, E. A., Gariepy, G., \& Greenfield, B. (2014). Cost-effectiveness of a rapid response team intervention for suicidal youth presenting at an emergency department. Canadian Journal of Psychiatry, 59(6), 310-318.

Lento, R. M., Ellis, T. E., Hinnant, B. J., \& Jobes, D. A. (2013). Using the Suicide Index Score to predict treatment outcomes among psychiatric inpatients. Suicide and Life-Threatening Behavior, 43(5), 547-561. doi: 10.1111/sltb.12038

Linehan, M., Comtois, K. A., Murray, A. M., Brown, M.Z., Gallop, R. J., et al. (2006). Two-year randomized controlled trial and follow-up of Dialectical Behavior Therapy vs. therapy by experts for suicidal behaviors and Borderline Personality Disorder. Archives of General Psychiatry, 63 (7), 757-766. doi: 10.1001/archpsyc.63.7.757

Marty, M. A., Segal, D. L., Coolidge, F. L., \& Klebe, K. J. (2012). Analysis of the psychometric properties of the Interpersonal Needs Questionnaire (INQ) among community-dwelling older adults. Journal of Clinical Psychology, 68(9), 10081018. doi: $10.1002 /$ jclp. 21877

Maslow, A. H. (1943). A theory of human motivation. Psychological Review, 50, 370396.

Mazzucchelli, T., Kane, R., \& Rees, C. (2009). Behavioral activation treatments for depression in adults: A meta-analysis and review. Clinical Psychological Science and Practice, 16, 383-411. doi: 10.1111/j.1468-2850.2009.01178.x

Mehlum L., Tormoen, A. J., Ramberg, M., Haga, E., Diep, L. M., Laberg, S., Larsson, B. S., Stanley, B. H., Miller, A. L., Sund, A. M., \& Groholt, B. (2014). Dialectical behavior therapy for adolescents with repeated suicidal and self-harming behavior: A randomized trial. Journal of the American Academy of Child \& Adolescent Psychiatry, 53(10), 1082-1091. 
Mental Health America of Texas. (2013). ASK and Prevent Suicide Retrieved 5/8/2014, from www.texassuicideprevention.org/information-library/app-promotions/\#ask

Merchant, C. (2010). An ecological exploration of black adolescent psychopathology: Depression and suicidal ideation Doctoral dissertation. Ann Arbor: University of Michigan.

Monteith, L. L., Menefee, D. S., Pettit, J. W., Leopoulos, W. L., \& Vincent, J. P. (2013). Examining the interpersonal psychological theory of suicide in an inpatient veteran sample. Suicide and Life-Threatening Behavior, 43(4), 418-428. doi: $10.1111 /$ sltb. 12027

Munoz, R. F., Mrazek, P. J., \& Haggerty, R. J. (1996). Institute of Medicine report on prevention of mental disorders: Summary and commentary. American Psychologist, 51(11), 1116-1122. doi: 10.1037/0003-066X.51.11.1116

Newman, D. A. (2003). Longitudinal modeling with randomly and systematically missing data: A simulation of ad hoc, maximum likelihood, and multiple imputation techniques. Organizational Research Methods, 6(3), 328-362. doi: $10.1177 / 1094428103254673$

O'Carroll, P., Berman, A., Maris, R., Moscicki, E., Tanney, B., \& Silverman, M. (1996). Beyond the Tower of Babel: A Nomenclature for Suicidology. Suicide and LifeThreatening Behavior, 26(3), 237-252. doi: 10.1111/j.1943.278X.1996.tb00609.x

O'Keefe, V. M., \& Wingate, L. R. (2013). The role of hope and optimism in suicide risk for American Indians/Alaskan Natives. Suicide and Life-Threatening Behavior, 43(6), 621-633. doi: 10.1111/sltb.12044

O'Keefe, V. M., Wingate, L. R., Tucker, R. P., Rhoades-Kerswill, S., Slish, M. L., \& Davidson, C. L. (2014). Interpersonal suicide risk for American Indians: Investigating thwarted belongingness and perceived burdensomeness. Cultural Diversity and Ethnic Minority Psychology, 20(1), 61-67. doi: 10.1037/a0033540

Ohman, A., \& Mineka, S. (2001). Fears, phobias, and preparedness: Toward an evolved module of fear and fear learning. Psychological Review, 108(3), 483-522. doi: 10.1037/0033-295X.108.3.483

Pettit, J., Green, K. L., Grover, K., Schatte, D., \& Morgan, S. (2011). Domains of chronic stress and suicidal behaviors among inpatient adolescents. Journal of Clinical Child and Adolescent Psychology, 40(3), 494-499. doi: $10.1080 / 15374416.2011 .563466$ 
Pettit, J. W., Lam, A. G., Voelz, Z. R., Walker, R. L., Perez, M., Joiner, T. E., . . He, Z. (2002). Perceived burdensomeness and lethality o suicide method among suicide completers in the People's Republic of China. Omega, 45(1), 57-67. doi: 10.2190/15LA-0WCR-N0DM-BWT8

Phillips, M. R., Yang, G., Zhang, Y., Wang, L., Ji, H., \& Zhou, M. (2002). Risk factors for suicide in China: A national case-control psychological autopsy study. The Lancet, 360, 1728-1736.

Pluzinski, C. \& Qualls, W. J. (1986). Consumer response to marketing stimuli: The relationship between affect, cognition, and behavior. Advances in Consumer Research, 13, 231-234.

Proulx, F., Lesage, A. D., \& Grunberg, F. (1997). One hundred in-patient suicides. The British Journal of Psychiatry, 171 (3), 247-250. doi: 10.1192/bjp.171.3.247

Quinnett, P. G. (1995). QPR for suicide prevention. Spokane, WA: QPR Institute Inc.

Rasmussen, K. A., \& Wingate, L. R. (2011). The role of optimism in the interpersonalpsychological theory of suicidal behavior. Suicide and Life-Threatening Behavior, 41(2), 137-148. doi: 10.1037/0022-3514.88.4.589

Rasmussen, K. A., Slish, M. L., Wingate, L. R., Davidson, C. L., \& Grant, D. M. (2012). Can perceived burdensomeness explain the relationship between suicide and perfectionism? Suicide and Life-Threatening Behavior, 42(2), 121-128. doi: 10.1111/j.1943-278X.2011.00074.X

Rathus, J. H., \& Miller, A. L. (2002). Dialectical Behavior Therapy adapted for suicidal adolescents. Suicide and Life-Threatening Behavior, 32(2), 146-157. doi: 10.1521/suli.32.2.146.24399

Rausch, J. R., Maxwell, S. E., \& Kelley, K. (2003). Analytic methods for questions pertaining to a randomized pretest, posttest, follow-up design. Journal of Clinical Child \& Adolescent Psychology, 32(3), 467-486. doi: 10.1207/S15374424JCCP3203 15

Reimer, M., Athay, m. M., Bickman, L., Breda, C., Kelley, S. D., \& Vides de Andrade, A. R. (2012). The Peabody Treatment Progress Battery: History and methods for developing a comprehensive measurement battery for youth mental health. Administration and Policy in Mental Health and Mental Health Services Research, 39(1-2), 3-12. doi: 10.1007/s10488-012-0404-1

Robinson, J., Hetrick, S., Cox, G., Bendall, S., Yung, Al., Yuen, H. P., Templer, K., \& Pirkis, J. (2014). The development of a randomized controlled trial testing the effects of an online intervention among school students at risk for suicide. $M B C$ Psychiatry, 14(155), 1-9. doi: 10.1186/1471-244X/14/155 
Robinson, J., Hetrick, S., Gook, S., Cosgrave, E., Yuen, H. P., McGorry, P., \& Yung, A. (2009). Study protocol: The development of a randomised controlled trial testing a postcard intervention designed to reduce suicide risk among young help-seekers. BMC Psychiatry, 9(59), 1-6. doi: 10.1186/1471-244x-9-59

Robinson, J., Hetrick, S. E., \& Martin, C. (2011). Preventing suicide in young people: Systematic review. Australian and New Zealand Journal of Psychiatry, 45(1), 326. doi: 10.3109/00048674.2010.511147.

Rorsman, B. (1973). Suicide in psychiatric inpatients: A comparative study. Social Psychiatry, 8 (2), 55-66.

Rounsaville, B. J., Carroll, K. M., \& Onken, L. S. (2001). A stage model of behavioral therapies research: Getting started and moving on from stage I. Clinical Psychology: Science and Practice, 8(2), 133-142. doi: 10.1093/clipsy.8.2.133

Sabbath, J. C. (1969). The suicidal adolescent - the expendable child. Journal for the American Academy of Adolescent Psychiatry, 8(2), 272-289. doi: 10.1016/S00027138(09)61906-3

Shneidman, E. S. (1993). Commentary: Suicide as psychache. Journal of Nervous and Mental Disease, 181(3), 145-147.

Shneidman, E. S. (1998). Further reflections on suicide and psychache. Suicide and LifeThreatening Behavior, 28(3), 245-250. doi: 10.1111/j.1943-278X.1998.tb00854.x

Silverman, M. M., Berman, A. L., Sanddal, N. D., O'Carroll, P. W., \& Joiner, T. E. (2007). Rebuilding the Tower of Babel: A revised nomenclature for the study of suicide and suicidal behaviors: Part II: Suicide-related ideations, communications and behaviors. Suicide and Life-Threatening Behavior, 37(3), 264-264-277. doi: 10.1521/suli.2007.37.3.264

Substance Abuse and Mental Health Services Administration. (2014). National Suicide Prevention Lifeline Retrieved June 5, 2014, from www.suicidepreventionlifeline.org

Suicide Prevention Resource Center and SPAN USA. (2010). Charting the future of suicide prevention: A 2010 progress review of the national strategy and recommendations for the decade ahead. Newton, MA: Education Development Center, INC.

Tabachnick, B. G. \& Fidell, L. S. (2013). Using multivariate statistics, $6^{\text {th }}$ edition. Pearson Education Inc., Boston, MA. 
Taliaferro, L. A., \& Muehlenkamp, J. J. (2014). Risk and protective factors that distinguish adolescents who attempt suicide from those who only consider suicide in the past year. Suicide and Life-Threatening Behavior, 44(1), 6-22. doi: 10.1111/sltb.12046

The Trevor Project. (2015). The Trevor Project. Retrieved 1/26/2015. www.thetrevorproject.org.

Thompson, E. A., Eggert, L., Randell, B., \& Pike, K. (2001). Evaluation of indicated suicide risk prevention approaches for potential high school dropouts. American Journal of Public Health, 91(5), 742-752.

Townsend, K. C., \& McWhirter, B. T. (2005). Connectedness: A review of the literature with implications for counseling, assessment, and research. Journal of Counseling and Development, 83, 191-201. doi: 10.1002/j.1556-6678.2005.tb00596.x

Troister, T., Davis, M. P., Lowndes, A., \& Holden, R. R. (2013). A five-month longitudinal study of psychache and suicidal ideation: Replication in general and high-risk university students. Suicide and Life-Threatening Behavior, 43(6), 611620. doi: $10.1111 /$ sltb. 12043

Tucker, R. P., Wingate, L. R., O'Keefe, V. M., Slish, M. L., Judah, M. R., \& RhoadesKerswill, S. (2013). The moderating effect of humor style on the relationship between interpersonal predictors of suicide and suicidal ideation. Personality and Individual Differences, 54, 610-615. doi: 10.1016/j.paid.2012.11.023

United States Department of Health and Human Services. (2001). National strategy for suicide prevention: Goals and objectives for action. Rockville, MD.

United States Department of Health and Human Services. (1999). Surgeon General's Call to Action to Prevent Suicide. Rockville, MD.

van der Feltz-Cornelis, C. M., Sarchiapone, M., Postuvan, V., Volker, D., Roskar, S., Grum, A. T., et al. (2011). Best practice elements of multilevel suicide prevention strategies: A review of systematic reviews. Crisis, 32(6), 319-333. doi: $10.1027 / \mathrm{a} 000001$

Van Orden, K. A. (2009). Construct validity of the Interpersonal Needs Questionnaire. (Unpublished doctoral dissertation). Florida State University, Tallahassee, FL.

Van Orden, K. A., Cukrowicz, K. C., Witte, T. K., \& Joiner, T. E. (2012). Thwarted belongingness and perceived burdensomeness: Construct validity and psychometric properties of the Interpersonal Needs Questionnaire. Psychological Assessment, 24(1), 197-215. doi: 10.1037/a0025358 
Van Orden, K. A., Witte, T. K., Cukrowicz, K. C., Braithwaite, S. R., Selby, E. A., \& Joiner, T. E. (2010). The interpersonal theory of suicide. Psychological Review, 117(2), 575-600. doi: 10.1037/0033-295x.96.2.358

Van Orden, K. A., Witte, T., Gordon, K., Bender, T., \& Joiner, T. (2008). Suicidal desire and the capability for suicide: Tests of the interpersonal-psychological theory of suicidal behavior among adults. Journal of Consulting and Clinical Psychology, 76, 72-83. doi: 10.1037/0022-006x.76.1.72

Wong, Y. J., Koo, K., Tran, K. K., Chiu, Y.-C., \& Mok, Y. (2011). Asian American college students' suicide ideation: A mixed-methods study. Journal of Counseling Psychology, 58(2), 197-209. doi: 10.1037/0022-006x.67.6.100410596523

Wyman, P. A., Brown, C. H., Inman, J., Cross, W., Schmeelk-Cone, K., Guo, J., \& Pena, J. B. (2008). Randomized trial of a gatekeeper program for suicide prevention: 1year impact on secondary school staff. Journal of Consulting and Clinical Psychology, 76(1), 104-115. doi: 10.1037/0022-006x.76.1.104

Wyman, P. A., Brown, C. H., LoMurray, M., Schmeelk-Cone, K., Petrova, M., Yu, Q., ... Wang, W. (2010). An outcome evaluation of the Sources of Strength suicide prevention program delivered by adolescent peer leaders in high schools. American Journal of Public Health, 100(1), 1653-1661. doi: 10.2105/ajph.2009.190025

Ybarra, M. L., \& Eaton, W. W. (2005). Internet-based mental health interventions. Mental Health Services Research, 7(2), 75-87. doi: 10.1007/s11020-005-3779-8

Zenere, F. J., \& Lazarus, P. J. (1997). The decline of youth suicidal behavior in an urban, multicultural public school system following the introduction of a suicide prevention and intervention program. Suicide and Life-Threatening Behavior, 27(4), 397-403. doi: 10.1111/j.1943-278X.1997.tb00518.x 
VITA

\section{RYAN HILL}

2005-2009

B.A., Psychology

University of Michigan

Ann Arbor, Michigan

2010-2015

M.S., Psychology

Doctoral Candidate

Florida International University

Miami, Florida

\section{PUBLICATIONS AND PRESENTATIONS}

Hill, R. M., Castellanos, D. A., \& Pettit, J. W. (2011). Suicidal behavior and anxiety in children and adolescents: A review of the past 20 years. Clinical Psychology Review, 31, 1133-1144. doi: 10.1016/j.cpr.2011.07.008

Hill, R. M. \& Pettit, J. W. (in press). Perceived burdensomeness and suicide-related behaviors: Current evidence and future directions. Journal of Clinical Psychology. doi: $10.1002 /$ jclp. 22071

Hill, R. M. \& Pettit, J. W. (2013). The role of autonomy needs in suicidal ideation: Integrating the interpersonal-psychological theory of suicide and self-determination theory. Archives of Suicide Research, 17(3), 288-301, doi:

10.1080/13811118.2013.777001

Hill, R. M. \& Pettit, J. W. (2012). Suicidal ideation and sexual orientation in college students: The roles of perceived burdensomeness, thwarted belongingness, and perceived rejection due to sexual orientation. Suicide and Life-Threatening Behavior, 42(5), 567579, doi: 10.1111/j.1943-278X.2012.00113.x

Hill, R. M., Pettit, J. W., Green, K. L., Morgan, S. T., \& Schatte, D. J. (2012). Precipitating events in adolescent suicidal crises: Exploring stress-reactive and nonreactive risk processes. Suicide and Life-Threatening Behavior, 42(1), 11-21, doi: 10.1111/j.1943-278X.2011.00067.x

Hill, R. M., Pettit, J.W., Lewinsohn, P.M., Seeley, J.R., \& Klein, D. N. (in press). Escalation to Major Depressive Disorder among adolescents with subthreshold depressive symptoms: Evidence of multiple risk pathways. Journal of Affective Disorders. doi: 10.1016/j.jad.2014.02.011

Hill, R. M., Rey, Y., Marin, C., Sharp, C., Green, K. L., \& Pettit, J. W. (in press). Evaluating the Interpersonal Needs Questionnaire: Comparison of the reliability, factor 
structure, and predictive validity across five versions. Suicide and Life-Threatening Behavior.

Hill, R. M., Yaroslavsky, I., \& Pettit, J. W. (in press). Enhancing depression screening to identify college students at risk for persistent depressive symptoms. Journal of Affective Disorders.

Horwitz, A., Hill, R. M., \& King, C. A. (2011). Specific coping behaviors in relation to adolescent depression and suicide ideation. Journal of Adolescence, 34, 1077-1085. doi:10.1016/j.adolescence.2010.10.004

Jacobson, C. M., Hill, R. M., Petit, J., \& Grozeva, D. (in press). The association of interpersonal and intrapersonal emotional experiences with non-suicidal self-injury among young adults. Archives of Suicide Research.

Jacobson, C. M., Hill, R. M., Pettit, J., \& Miranda, R. (in press). The Measure of Verbally Expressed Emotion: Development and factor structure of a scale designed to assess comfort expressing feelings to others. Journal of Psychopathology and Behavioral Assessment.

King, C. A., Hill, R. M., Wynne, H., \& Cunningham, R. (2012). Adolescent suicide risk screening: The effect of communication about type of follow-up on adolescents' responses. Journal of Clinical Child and Adolescent Psychology, 41(4), 508-515, doi: 10.1080/15374416.2012.680188

Meinzer, M. C., Hill, R. M., \& Pettit, J. W. (in press). Exploring the relationship between ADHD symptoms and depressive symptoms in college students: The role of family support. Journal of Psychopathology and Behavioral Assessment.

Meinzer, M.C., Pettit, J.W., Leventhal, A.M., \& Hill, R. M. (2012). Explaining the covariance between Attention-Deficit Hyperactivity Disorder symptoms and depressive symptoms: The role of reward responsivity. Journal of Clinical Psychology, 68(10), 1111-1121, doi: 10.1002/jclp.21884

O’Mara, R., Hill, R. M., Cunningham, R., \& King, C. A. (2012). Adolescent and parent attitudes toward screening for suicide risk and mental health problems in the pediatric emergency department. Pediatric Emergency Care, 28(7), 626-632. doi:

10.1097/PEC.0b013e31825cfb10 